\title{
Methodological aspects of deep brain stimulation
}

Citation for published version (APA):

Alptekin, O. (2021). Methodological aspects of deep brain stimulation: the untold story behind DBS surgery. [Doctoral Thesis, Maastricht University]. Maastricht University. https://doi.org/10.26481/dis.20210618oa

Document status and date:

Published: 01/01/2021

DOI:

10.26481/dis.20210618oa

Document Version:

Publisher's PDF, also known as Version of record

\section{Please check the document version of this publication:}

- A submitted manuscript is the version of the article upon submission and before peer-review. There can be important differences between the submitted version and the official published version of record.

People interested in the research are advised to contact the author for the final version of the publication, or visit the DOI to the publisher's website.

- The final author version and the galley proof are versions of the publication after peer review.

- The final published version features the final layout of the paper including the volume, issue and page numbers.

Link to publication

\footnotetext{
General rights rights.

- You may freely distribute the URL identifying the publication in the public portal. please follow below link for the End User Agreement:

www.umlib.nl/taverne-license

Take down policy

If you believe that this document breaches copyright please contact us at:

repository@maastrichtuniversity.nl

providing details and we will investigate your claim.
}

Copyright and moral rights for the publications made accessible in the public portal are retained by the authors and/or other copyright owners and it is a condition of accessing publications that users recognise and abide by the legal requirements associated with these

- Users may download and print one copy of any publication from the public portal for the purpose of private study or research.

- You may not further distribute the material or use it for any profit-making activity or commercial gain

If the publication is distributed under the terms of Article $25 \mathrm{fa}$ of the Dutch Copyright Act, indicated by the "Taverne" license above, 
Methodological Aspects of Deep Brain Stimulation: The Untold Story Behind DBS Surgery

Onur Alptekin 



\section{Statements}

\section{Belonging to the PhD Thesis}

\section{Methodological Aspects of Deep Brain Stimulation: The Untold Story Behind DBS Surgery}

\section{Onur Alptekin}

1. The road to success in DBS therapy passes through the ability to cope with surgical and technical complications. (This Thesis).

2. The error sources in stereotactic DBS procedures that are linked to the human hand sensitivity have not been a serious research subject until today. (This Thesis).

3. To reach a high level of precision in stereotactic surgeries, there are several sources of potential error which should be taken into account. (This Thesis).

4. With MER, alternative trajectories are immediately available. The trajectory with the second longest and, if needed, the third longest STN activity can be used as alternative trajectories. (This Thesis).

5. MER procedure may be more valuable for the permanent electrode implantation of second side of a bilateral DBS surgery than the first side in order to compensate error sources that might arise intraoperatively. (This Thesis).

6. The success of the therapy relies on three main factors: the appropriate selection of patients, the accurate placement of the DBS lead in the sensorimotor regions of the target nuclei, and optimal choice of electrical parameters for stimulation. (William J. Marks, Jr)

7. Neurons that fire together, wire together. (Donald Olding Hebb)

8. We are just an advanced breed of monkeys on a minor planet of a very average star. But we can understand the Universe. That makes us something very special. (Stephen William Hawking)

9. I hated every minute of training, but I said, 'Don't quit. Suffer now and live the rest of your life as a champion. (Muhammad Ali)

10. Awizard is never late, nor is he early, he arrives precisely when he means to. (John Ronald Reuel Tolkien) 


\section{Cover:}

The cover illustrates and refers to one of the key messages of this thesis. The term butterfly effect comes from an analogy, from Chaos theory, where a butterfly flaps its wings somewhere in the world and a tornado occurs in a different location. Likewise, sub-millimetric factors in early stages of DBS surgeries might contribute the outcome of the complete therapy. The illustrated butterfly has basal ganglia patterns on its wings. Black and white half of the butterfly refers to radiological imaging techniques and image fusion.

(C) Onur Alptekin 2021

Methodological Aspects of Deep Brain Stimulation: The Untold Story Behind DBS Surgery.

All rights are reserved. No part of this book may be reproduced or transmitted in any form or by any means, without permission in writing from the copyright holder.

ISBN: 987-625-409-526-9

Cover Design: Onur Alptekin

Layout and Production: Hızlı Kopyalama Ajans ve Bas. Hiz. Tic. Ltd. Şti. I www.hizlikopyalama.com 


\title{
Methodological Aspects of Deep Brain Stimulation: The Untold Story Behind DBS Surgery
}

\author{
Dissertation \\ To obtain the degree of Doctor at Maastricht University, \\ on the authority of the Rector Magnificus Prof. dr. Rianne M. Letschert \\ in accordance with the decision of the Board of Deans, \\ to be defended in public on Friday 18 June 2021, at 10:00 hours
}

by

Onur Alptekin

Born in Ankara (Turkey) on $20^{\text {th }}$ of August 1984 


\section{Supervisor}

Prof. Dr. Y. Temel

\section{Co-supervisors}

Dr. L. Ackermans

Dr. E. Kocabicak (Samsun, Turkey)

\section{Assessment Committee}

Prof. Dr. J. van Zundert (Chairman)

Prof. Dr. H. van Santbrink

Prof. Dr. T. Uçar (Antalya, Turkey)

Dr. A. Jahanshahianvar

Dr. S. Tan (Univ. of Aachen, Germany) 
To my mother Ülker Alptekin and father Tahsin Alptekin. .... and to my wife Didem Savaş Alptekin 


\section{Table of Contents}

Chapter 1

Introduction

Chapter 2

Perioperative Technical Complications In Deep Brain Stimulation Surgeries

Alptekin, O., Kocabicak, E., Gubler, F. S., Ackermans, L., Kubben, P. L., Temel, Y. (2018). Perioperative Technical Complications in Deep Brain Stimulation Surgeries. Turkish neurosurgery, 28(3), 483-489.

Chapter 3

Stereotactic Accuracy and Frame Mounting; A Phantom Study

Alptekin, O., Gubler, F. S., Ackermans, L., Kubben, P. L., Kuijf, M. L., Kocabicak, E., Temel, Y. (2019). Stereotactic accuracy and frame mounting: A phantom study. Surgical Neurology International, 10.

Chapter 4

Impact of Computed Tomography Image Slice Thickness on Stereotaxy a Phantom Study

Alptekin, O., Gubler, F. S.,Ackermans L., Kubben P.L., Kuijf, M.L., Kocabicak, E., Temel, Y.

Chapter 5

Is There Still Need for Microelectrode Recording Now The Subthalamic Nucleus Can Be Well Visualized With High Field and Ultrahigh MR Imaging?

Kocabicak, E., Alptekin, O., Ackermans, L., Kubben, P., Kuijf, M., Kurt, E., Esselink, R., Temel, Y. (2015). Is there still need for microelectrode recording now the subthalamic nucleus can be well visualized with high field and ultrahigh MR imaging? Frontiers in integrative neuroscience, 9, 46.

Chapter 6

Microelectrode Recording for Deep Brain Stimulation of the Subthalamic Nucleus in Patients with Advanced Parkinson's Disease: Advantage or Loss of Time?

Alptekin, O., Kocabicak, E., Aygun, D., Yildiz, O., Temel, Y. (2019). Microelectrode Recording for Deep Brain Stimulation of the Subthalamic Nucleus in Patients with Advanced Parkinson's Disease: Advantage or Loss of Time? Turkish Neurosurgery, 29(5), 677-682. 
Impact

Acknowledgements

Curriculum Vitae 

Chapter 1

Introduction 


\section{Introduction}

Deep brain stimulation (DBS) is a surgical therapy used in the treatment of several neurological disorders such as Parkinson's Disease (PD), essential tremor (ET), dystonia, epilepsy, and psychiatric disorders including obsessive-compulsive disorder and depression ${ }^{1-6}$. Today, DBS therapy has become a widely applied procedure throughout the world and has largely replaced ablative surgery for neurological and psychiatric diseases ${ }^{7}$.

Since the introduction of DBS therapy clinically, several individual technologies have been used in order to locate the implantable electrodes in the desired anatomical targets. Stereotactic frames were the fundamental surgical instruments for DBS therapy and were evolved for daily requirements. In the early years, radiological imaging needs were met with ventriculography ${ }^{8}$. Then, the imaging technique was evolved to computed tomography (CT) and later to magnetic resonance imaging (MRI). With the advances in radiological imaging technologies, stereotactic measurements on radiological films were abandoned and stereotactic planning softwares were developed in order to ease the surgeon's approach for anatomical targeting. Electrophysiological recording devices were developed and used with the purpose of examining neurophysiological activities and investigating the effects/side effects of electrical stimulation of related anatomical structures within the brain, which is called macrostimulation. Additionally, these devices were further developed in order to perform and analyze local field potential (LFP) recordings. All these elaborated technologies and developments have led the DBS surgical protocols to evolve and shape the golden standards of the surgical application of DBS therapy ${ }^{9,10}$.

Today, surgical instruments and technologies provide countless important advantages for surgical teams during DBS electrode implantation. Anatomical brain nuclei within the scope of DBS surgery can easily be detected and located with detailed high definition radiological images ${ }^{11,12}$. Stereotactic planning stations have the capability to perform image fusion process with high speed and accuracy which makes surgical targeting and stereotactic planning easier. Today, electrophysiological recording devices are not only used to analyze and process signals but also can generate three-dimensional (3D) models of the investigated brain nuclei from the neurophysiological signals ${ }^{13}$.

However, in order to have successful results with DBS therapy, all these technologies and instruments must be used in harmony. Incompatibilities between surgical instruments/systems or errors due to lack of user experience or information can decrease the success rate of the therapy. With respect to all these described complexities, sceptic thoughts have arisen on the steps of DBS surgery and surgical procedure steps were scientifically questioned on different platforms.

To date, complications related to surgical approach and implantable products/materials of DBS therapy have been discussed and reported in several studies ${ }^{14}$. These surgical complications of DBS surgeries are clearly known and can be managed in most cases ${ }^{15}$. However, there is very limited information on technical complications linked to surgical instruments/systems and this issue has not been investigated in details. Discussion of surgical instruments/systems related to complications seems to be a must for managing these complications before they arise. 
Stereotactic frames and planning stations are the most crucial surgical instruments/systems for the application of DBS surgery. These systems allow surgeons to work in sub-millimeter precision to reach deeply located anatomical structures in the brain. Although users intend to use these systems with recommended standards which are defined by the manufacturers, the weakness of human hand sensitivity in sub-millimetric tasks has always been an important issue. Today, stereotactic frames are mounted to patient heads under stressful conditions. Moreover, stereotactic coordinate calculations can still be performed manually by using the calculation methods described in the literature without planning stations. The error sources in stereotactic DBS procedures that are linked to the human hand sensitivity have not been a serious research subject until today.

Indispensably, radiological information is required in DBS procedures with the purpose of preoperative planning of the target nuclei in the brain and examining the quality of the positioned lead and its anatomical correlation $^{16}$. MRI technology has provided important advantages for the visualization of the anatomic structures in the brain and is still the best radiological imaging option for stereotactic procedures. However, CT is still a good option to extract stereotactic information due to some important advantages it has. Stereotactic planning stations combine MR and CT images with a software process called image fusion to complement the shortcomings of two independent image groups of the same patient. Due to the image quality and resolution advantages in soft tissues, MR images are mostly preferred for targeting brain nuclei in DBS surgeries. Many articles have been published on MRI of brain nuclei within the scope of DBS therapy which detailed the imaging standards for different MR devices and sequences ${ }^{17,18}$. Within the MR-CT image fusion concept, CT images are mostly preferred for extracting the stereotactic coordinates of predefined anatomic targets ${ }^{19}$. However, the information related to CT imaging standards is very limited and mostly defined by stereotactic instrument manufacturers. Technically, stereotactic CT imaging can be performed at various slice thickness rates within the range between $0.01 \mathrm{~mm}$ and $5 \mathrm{~mm}$. However, as the slice thickness of the CT series decreases, the image processing load of the stereotactic planning station and the amount of $x$-ray that the patient is exposed to increase inversely ${ }^{20}$. At this point, investigation of very low-thickness stereotactic CT image usage and the optimal slice thickness parameters for stereotactic CT imaging will prove valuable results.

Electrophysiology has made important contribution to DBS therapy in both clinical applications and preclinical studies. Today, neurophysiological activity patterns of brain nuclei within the scope of DBS therapy are wellknown and these electrophysiological patterns guide the surgeon to determine the anatomic position of the permanent electrodes that will be implanted during DBS surgeries ${ }^{21,22}$.

Advances in surgical equipment technology have also led to the questioning of the systems that are used to obtain intraoperative neurophysiological signals in DBS surgeries. Microelectrode recording (MER) is an intraoperative electrophysiological signal recording method in DBS surgeries which is used to obtain and investigate the neurophysiological signal patterns of the brain nuclei. Macro stimulation is mostly performed to investigate the effects and side effects of acute stimulation intra-operatively at the neural activity location which is determined by $\mathrm{MER}^{23}$. However, microelectrodes used during MER and macrostimulation applications have initiated many controversies due to the surgical complications and the risks that their physical structures can pose $\mathrm{e}^{24,25}$. Moreover, the time spent for MER and macrostimulation and the benefits of these applications are often discussed ${ }^{26-30}$. 


\section{Aim of the present study}

In this study, we presented and questioned the factors that contribute to the DBS surgery from technical perspective through five different aspects. In chapter 2, we presented the retrospective qualitative analysis of complications from technical instruments/systems which were experienced during implantation of 921 DBS electrodes in our centers. In chapter 3, we reported the relationship between stereotactic accuracy and stereotactic frame mounting through three different frame mounting scenarios with a phantom study. In this section, we investigated the effect of asymmetrically performed stereotactic frame mounting on DBS electrode implantation accuracy. In chapter 4, we investigated the effect of CT slice thickness on stereotactic calculations. We analyzed this factor for most frequently preferred slice thickness parameters used in clinical applications within the range between $0,5 \mathrm{~mm}$ and $5 \mathrm{~mm}$ with another phantom study. In chapter 5, we evaluated the requirement for MER use in DBS surgeries for PD patients, despite the advantages of MRI technology serving with high definition and resolution radiological images for soft tissues. In chapter 6, we discussed the role and the advantages of MER in DBS surgery of Parkinson's disease patients and the time-wise effect of MER in DBS surgeries. Finally in chapter 7, we presented an overall conclusion of our work. 


\section{References}

1. Volkmann J. Deep brain stimulation for Parkinson's disease. Parkinsonism \& related disorders. 2007;13:S462-S5.

2. Sandoe C, Krishna V, Basha D, et al. Predictors of deep brain stimulation outcome in tremor patients. Brain stimulation.2018;11(3):592-9.

3. Krauss JK, Yianni J, Loher TJ, Aziz TZ. Deep brain stimulation for dystonia. Journal of Clinical Neurophysiology. 2004;21(1):18-30.

4. Van Der Vlis TAB, Schijns OE, Schaper FL, et al. Deep brain stimulation of the anterior nucleus of the thalamus for drug-resistant epilepsy. Neurosurgical review. 2019;42(2):287-96.

5. Alonso P, Cuadras D, Gabriëls L, et al. Deep brain stimulation for obsessive-compulsive disorder: a meta-analysis of treatment outcome and predictors of response. PLoS One. 2015;10(7).

6. Zhou C, Zhang H, Qin Y, et al. A systematic review and meta-analysis of deep brain stimulation in treatment-resistant depression. Progress in Neuro-Psychopharmacology and Biological Psychiatry. 2018;82:224-32.

7. Fox SH, Katzenschlager R, Lim SY, et al. International Parkinson and Movement Disorder Society evidence-based medicine review: update on treatments for the motor symptoms of Parkinson's disease. Movement Disorders. 2018;33(8):1248-66.

8. Lozano AM, Gildenberg PL, Tasker RR. Textbook of stereotactic and functional neurosurgery: Springer Science \& Business Media; 2009.

9. Kocabicak E, Temel Y. Deep brain stimulation of the subthalamic nucleus in Parkinson's disease: surgical technique, tips, tricks and complications. Clinical neurology and neurosurgery. 2013;115(11):2318-23.

10. Kühn AA, Volkmann J. Innovations in deep brain stimulation methodology. Movement Disorders. 2017;32(1):11-9.

11. Longhi M, Ricciardi G, Tommasi G, et al. The role of $3 \mathrm{~T}$ magnetic resonance imaging for targeting the human subthalamic nucleus in deep brain stimulation for Parkinson disease. Journal of Neurological Surgery Part A: Central European Neurosurgery. 2015;76(03):181-9.

12. Forstmann BU, Isaacs BR, Temel Y. Ultra high field MRI-guided deep brain stimulation. Trends in biotechnology. 2017;35(10):904-7.

13. Husch A, Gemmar P, Thunberg J, Hertel F, editors. Integration of sparse electrophysiological measurements with preoperative MRI using 3D surface estimation in deep brain stimulation surgery. Medical Imaging 2017: Image-Guided Procedures, Robotic Interventions, and Modeling; 2017: International Society for Optics and Photonics.

14. Moro E. Neurosurgery: Complications of DBS surgery - insights from large databases. Nature Reviews Neurology. 2016;12(11):617.

15. Fenoy AJ, Simpson RK. Risks of common complications in deep brain stimulation surgery: management and avoidance. Journal of neurosurgery. 2014;120(1):132-9.

16. Mirzadeh Z, Chapple K, Lambert M, Dhall R, Ponce FA. Validation of CT-MRI fusion for intraoperative assessment of stereotactic accuracy in DBS surgery. Movement Disorders. 2014;29(14):1788-95.

17. Geng D-y, Li Y-X, Zee C-S. Magnetic resonance imaging-based volumetric analysis of basal ganglia nuclei and substantia nigra in patients with Parkinson's disease. Neurosurgery. 2006;58(2):256-62.

18. Telford R, Vattoth S. MR anatomy of deep brain nuclei with special reference to specific diseases and deep brain stimulation localization. The neuroradiology journal. 2014;27(1):29-43.

19. Machado A, Rezai AR, Kopell BH, Gross RE, Sharan AD, Benabid AL. Deep brain stimulation for Parkinson's disease: surgical technique and perioperative management. Movement disorders: official journal of the Movement Disorder Society. 2006;21(S14):S247-S58.

20. Raman SP, Mahesh M, Blasko RV, Fishman EK. CT scan parameters and radiation dose: practical advice for radiologists. Journal of the American College of Radiology. 2013;10(11):840-6.

21. Urfalı B, Temel Y, Bergman H. Neurophysiology of the Basal Ganglia and Deep Brain Stimulation. Fundamentals and Clinics of Deep Brain Stimulation: Springer; 2020. p. 67-75.

22. Hutchison W, Allan R, Opitz H, et al. Neurophysiological identification of the subthalamic nucleus in surgery for Parkinson's disease. Annals of Neurology: Official Journal of the American Neurological Association and the Child Neurology Society. 1998;44(4):622-8.

23. Pollak P, Krack P, Fraix V, et al. Intraoperative micro-and macrostimulation of the subthalamic nucleus in Parkinson's disease. Movement disorders: official journal of the Movement Disorder Society. 2002;17(S3):S155-S61.

24. Xiaowu H, Xiufeng J, Xiaoping Z, et al. Risks of intracranial hemorrhage in patients with Parkinson's disease receiving deep brain stimulation and ablation. Parkinsonism \& related disorders. 2010;16(2):96-100.

25. Hariz MI. Safety and risk of microelectrode recording in surgery for movement disorders. Stereotactic and functional neurosurgery. 2002;78(3-4):146-57.

26. Kinfe TM, Vesper J. The impact of multichannel microelectrode recording (MER) in deep brain stimulation of the basal ganglia. Stereotactic and functional neurosurgery: Springer; 2013. p. 27-33.

27. McClelland III S. A cost analysis of intraoperative microelectrode recording during subthalamic stimulation for Parkinson's disease. Movement disorders. 2011;26(8):1422-7.

28. Bour LJ, Contarino MF, Foncke EM, et al. Long-term experience with intraoperative microrecording during DBS neurosurgery in STN and GPi. Acta neurochirurgica. 2010;152(12):2069-77.

29. Shamir RR, Zaidel A, Joskowicz L, Bergman H, Israel Z. Microelectrode recording duration and spatial density constraints for automatic targeting of the subthalamic nucleus. Stereotactic and functional neurosurgery. 2012;90(5):325-34.

30. Zeiler F, Wilkinson M, Krcek J. Subthalamic nucleus deep brain stimulation: an invaluable role for MER. Canadian journal of neurological sciences. 2013;40(4):572-5. 



\section{Chapter 2}

\section{Perioperative technical complications in deep brain stimulation surgeries}

Alptekin, O., Kocabicak, E., Gubler, F. S., Ackermans, L., Kubben, P. L., Temel, Y. (2018). Perioperative Technical Complications in Deep Brain Stimulation Surgeries.

Turkish neurosurgery, 28(3), 483-489. 


\section{Abstract}

Background: Deep brain stimulation(DBS) surgeries are multi-faceted and the various steps are interconnected. Since its first implementation, the method of DBS surgery has undergone changes. We have encountered several expected and also non-expected perioperative technical complications in the past seventeen years. Here, we describe the stereotactic frame, stereotactic localizer and planning station related complications and how we have managed them as much as possible.

Materials and Methods: This study is a retrospective qualitative analysis of the documented technical events encountered during DBS surgeries from 1999 onwards. We have collected these events from a cohort of approximately 921 DBS cases from the centers of the authors.

Results: Stereotactic frame related complications included movement-related fixation problems, head anatomy related problems, and lack of maintenance related issues. Localizer related complications were compatibility issues of the stereotactic localizer and planning station, field of view effect on fiducials, air bubbles in localizers using liquid solutions, and disengaged localizer effect. Planning station related complications included image fusion failures and CSF signal effect on image fusion.

Conclusions: The road to success in DBS therapy passes through the ability to cope with surgical and technical complications. Each step is unconditionally connected to the other, detection of the problems that can be encountered in advance and preparations for these negative conditions are the key to success for the group responsible for executing the therapy. We are still learning from these events and advance our surgical approaches. 


\section{Introduction}

Deep brain stimulation has become a widely applied procedure to treat patients with severe neurological and psychiatric disorders. This treatment modality is multi-faceted, and consists of several steps including patient selection, surgical planning of the anatomical target, surgical procedure, postoperative management and programming of the DBS therapy ${ }^{1}$. The short- and long-term therapeutic effects of DBS for various disorders have been documented extensively ${ }^{2,3}$. Although, the surgical ${ }^{4}$, hardware ${ }^{5}$ and target related complications of DBS surgery are better recognized and managed, technical complications of DBS related to surgical planning stations, surgical equipment, and implants are less well described. The authors have performed hundreds of cases (>900 DBS surgeries) in seventeen years. Since then, we have encountered many expected and unexpected, specific and general technical problems. Some of these technical problems were related to the stereotactic frame, stereotactic localizer and planning station. In this study, we describe the technical problems of DBS surgeries in detail and share our experience with these complications and their management for groups who are willing to start a DBS program or who have recently started. A detailed discussion of the surgery, and target related complications is beyond the scope of this article, and can be found elsewhere ${ }^{6,7}$.

\section{Stereotactic frame related complications}

In this section, we will discuss problems linked to the stereotactic frame, including movement-related fixation problems, head-anatomy related problems and lack of maintenance related problems.

\section{Movement-related fixation problems}

Mounting the stereotactic frame in patients with severe hyperkinetic movement disorders is a well-known challenge (Figure 1A). There are methods which can be helpful in these cases. One method is to apply ear fixation (Elekta, Stockholm, Sweden) (Figure 1B).

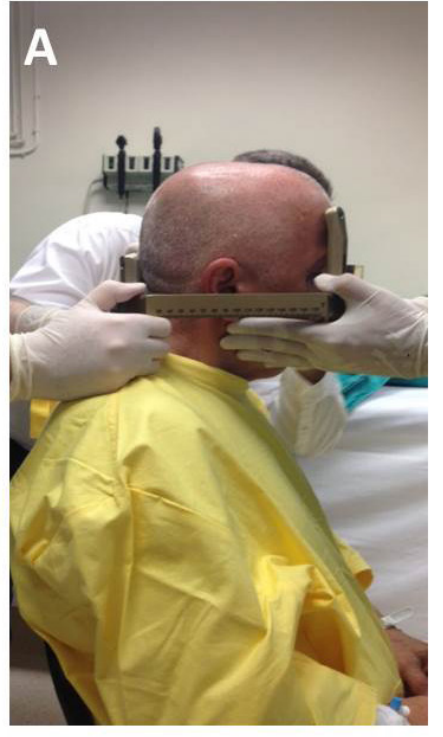

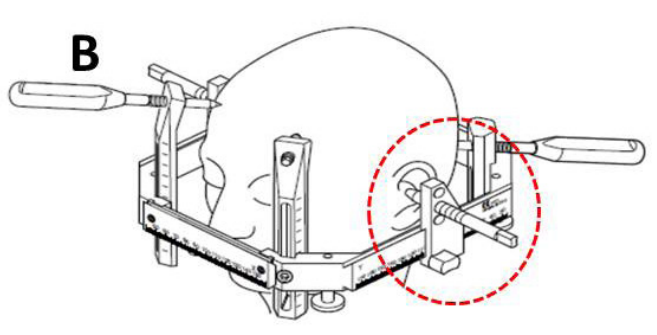

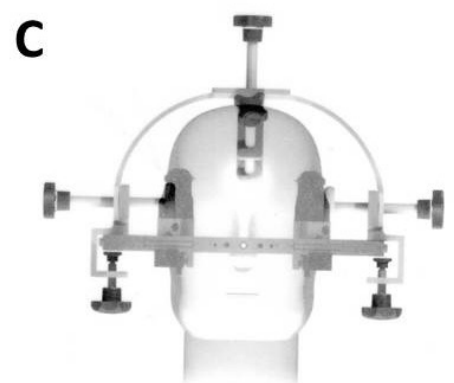

Figure 1:

This figure shows the three methods of mounting the stereotactic frame on the head of the patient. In figure $1 \mathrm{~A}$, the frame is fixated manually and mounted using the screws. In figure $1 \mathrm{~B}$, an ear-plug assisted fixation method and in figure $1 \mathrm{C}$ a head-device supported fixation method is illustrated.

An ear plug fits into the holder through one of its three holes. The hole that yields the required frame height with respect to the skull is used. The highest frame position is achieved by inserting the plugs into the lowest holes. After applying the ear plugs, the screws are tightened with a screwdriver to fixate the system to the head of the patient in the desired position. However, a serious issue is that this procedure can be very painful and cause substantial discomfort. In animal studies, usually a local anesthetic is applied to the ear ${ }^{8}$. Another and more recent method is the Stereotactic Position Aid device (Inomed, Emmendingen, Germany). The system enables the orientation of patient's head and frame before the frame screws are driven. This product can be used for the majority of the stereotactic frames and produces less discomfort as compared to ear fixation (Figure 1C). This complication has been seen in 7 out of 921 patients. 


\section{Head anatomy related problems}

Another problem can arise from the anatomy of the patient. The head anatomy is then not compatible with the shape of the frame. Especially the "arc support with slide parts" used in the Leksell Stereotactic G Frame and the Ark System (Elekta, Stockholm, Sweden) can be problematic in patients with a short-neck, even if the frame is placed as superiorly as possible (Figure 2A).

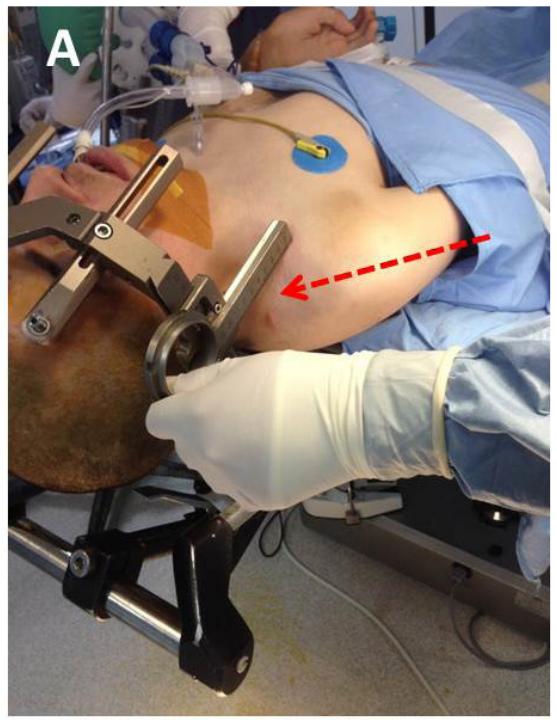

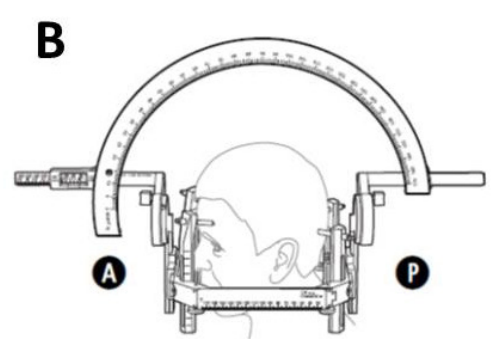

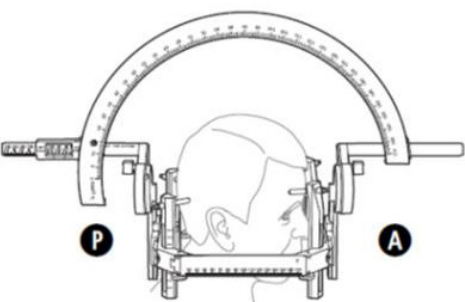

Figure 2:

Figure 2A illustrates the complication occurring with the Leksell G frame's slide parts in a patient with a dystonia with a short neck. The red dashed arrow points towards this anatomical obstacle. Figure 2B shows a solution for this problem by using a sagittal approach instead of a lateral one.

These parts may apply serious physical pressure to the shoulder and may result in stopping the surgery. An alternative approach would be to change the orientation from "lateral right" or "lateral left" to "sagittal anterior" or "sagittal posterior" orientations (Figure 2B). We think that inspection of the neck length before frame fixation is an important action which is likely to avoid this complication. One another option to manage this complication is fixing the frame as inferior as possible on the patient's head. With this kind of inferior fixation, the parts which make pressure to the shoulder will show lower $Z$ coordinate values. The parts causing pressure to the shoulder will stay away from the skin. In 1 patient of our series we have experienced this complication.

Another head anatomy related problem can be the frontal sinuses. Therefore, the anatomy of the frontal sinuses should be evaluated carefully before driving the frontal screws. If the screws are positioned slightly more superiorly in patients with a large frontal sinus, the outer layer can be fractured (Figure 3). This is a complication which may require surgical repair of the frontal sinus. This complication has been observed in 1 case.

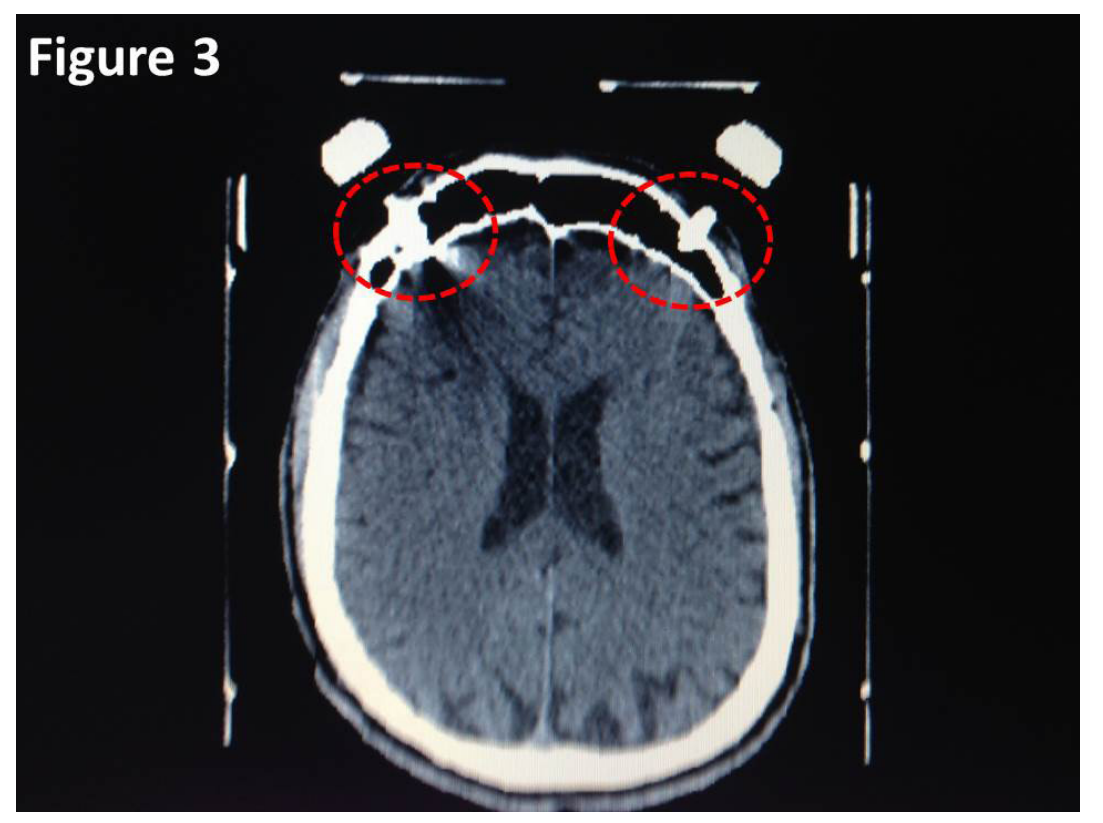

Figure 3:

Here, the frontal screws have penetrated the outer layer of the frontal sinus due to the combination of a superior placement of the screws and a large frontal sinus. 


\section{Lack of maintenance related problems}

Another often seen complication can be the inaccuracy of the stereotactic equipment due to wearing. An adequate maintenance, including periodic phantom-based checks and necessary calibrations, is essential to achieve millimetric precision in DBS surgeries. A well-known reason for deformation of the stereotactic equipment is trying to use it differently or use force against it. Loss of screws and small parts due to highpressure water applications is a frequently encountered drawback during the decontamination process of the stereotactic frames. A careful check of the integrity of the stereotactic equipment after the decontamination procedure is critical. In 14 cases of our series we experienced this complication.

\section{MRI/CT Localizer related complications}

In DBS surgeries, computed tomography (CT) and magnetic resonance imaging (MRI) technologies are used in order to transfer functional coordinates to the stereotactic coordinates. Image fusion features of planning stations are used for this conversion process. Stereotactic software used for surgical planning requires registration points called fiducials to perform 3-dimensional measurements. These reference points, appearing in white and circular or elliptic shape on radiological images, should be defined or registered to the stereotactic software either manually or automatically. This section describes the complications linked to the stereotactic localizers used during DBS procedures.

\section{Compatibility of Stereotactic Localizer and Planning Station}

Stereotactic planning softwares require different number of fiducial marks to be identified for different frame systems. For example, while Medtronic Framelink planning station (Medtronic, Minneapolis, USA) requires a minimum of 9 fiducials for the Leksell Stereotactic frame (Elekta, Stockholm, Sweden), 6 fiducial marks are sufficient for the Elekta SurgiPlan system (Elekta, Stockholm, Sweden). This means, if a surgical team has a localizer capable of providing 6 fiducial marks while they are working with a system requiring 9 fiducial points, it would be impossible to perform the targeting using these two systems. Although this point is logical, we have encountered problems, and therefore outline this problem here. In this sense, checking the compatibility of stereotactic localizer and stereotactic planning station before starting the measurement procedures is recommended. This complication has been seen in 1 case during our experience.

\section{Field of view effect on fiducials}

Another complication related with MRI/CT localizer is missing fiducials which has to be viewed together with the patient's anatomy on radiological images. If the technical personnel who perform the stereotactic MRI or CT imaging are not informed, they may create images without paying particular attention to the fiducial marks around the head. Images not including the required fiducial marks due to insufficient field of view (FOV) undermine the capability of stereotactic planning systems to carry out the desired image processing. On the other hand, applying an unnecessarily large FOV value may cause images with a very low resolution. In this respect, axial test imaging by having 1 or 2 slices on planned imaging area before total volume imaging will help to explore whether or not the FOV value is sufficient for the measurements. In 5 patients of our seris we have experienced this complication. 


\section{Air bubbles in localizers using liquid solutions}

In order to ensure fiducial marks are clearly noticed on MRI images, copper sulfate solution is recommended and used by some frame manufacturers. To obtain stereotactic MRI images for these systems, the localizer has to be filled with this solution. If the filling process is performed in an inadequate manner, air bubbles can remain in the copper sulfate solution filling localizer channels (Figure 4A).

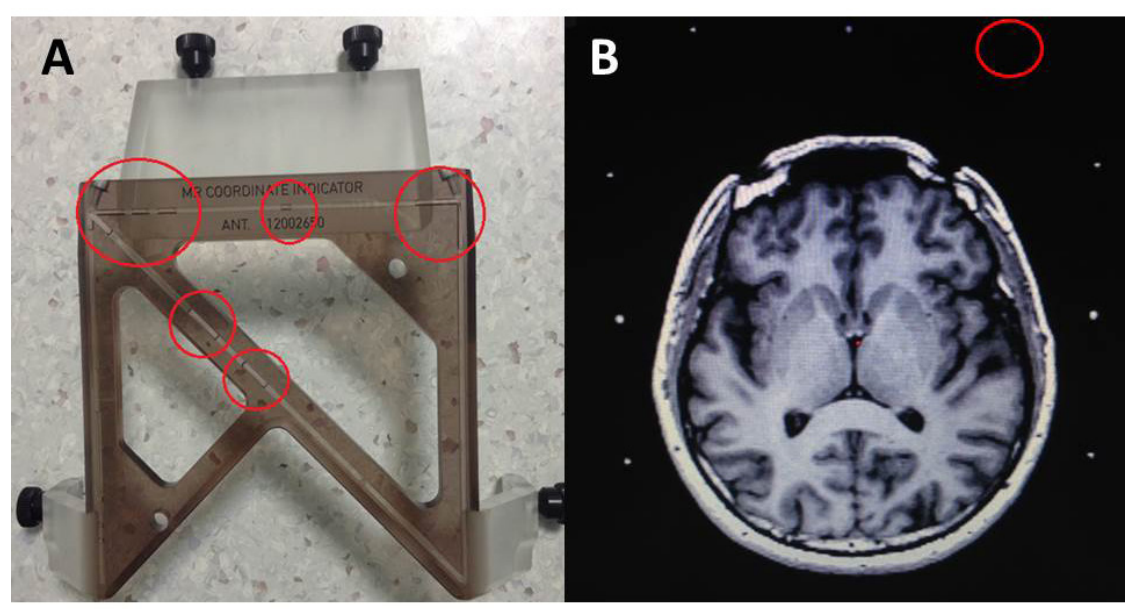

Figure 4:

Figure 4A shows the anterior part of a localizer filled with copper-sulfate. Red circles show the air bubbles in the channels of the localizer. In Figure 4B, a stereotactic T1 MR image is shown. The invisible fiducial is indicated with red circle on the radiological image.

Performing the imaging with air bubbles will result in poorly detectable fiducial marks (Figure 4B). In this case, if MRI is preferred for stereotactic imaging, filling the localizer channels or checking the contents of pre-filled channels of localizer, will be an effective way to prevent unwanted scenarios. This situation is not going to bring out any complication for the groups who are using CT localizers for stereotactic procedures. In 4 patients of our series, this complication was experienced.

\section{Disengaged localizer effect}

The engagement of the frame system and the localizer system is important to obtain accurate calculations before the DBS procedure. Independent of a CT or MRI localizer, it should be well attached and checked before the imaging process has been finalized. With images which are performed with a disengaged or not fully engaged localizer, the fiducials are not going to show the right points on the radiological images which will result in miscalculated targets. To avoid this complication, junction points between the stereotactic frame and stereotactic localizer must be checked if they are completely engaged. This miscalculated targeting will also affect the recording and the permanent implant quality as well. In our experience, we also experienced 1 misplaced electrode during postoperative imaging phase of the implant process because of this issue.

\section{Planning station related complications}

The goal of image fusion is to integrate complementary multi-sensor, multi-temporal and/or multi-view information into one new image ${ }^{9}$. Today, many different radiological image series are used for different purposes in the planning stages for DBS surgeries ${ }^{10-12}$. Some image series are meant to provide information about the vascular structures on the trajectories. Such information can be obtained using MRI and CT-images enhanced with contrast-agents ${ }^{13}$. Image fusion technology helps to integrate these two distinct pieces of information to serve an integrated purpose.

\section{Image fusion failures}

Although image fusion is often regarded as a software operation performed computer-based, the success and accuracy of the fusion depends on the imaging protocols. The way to make this process more precise is to ensure that imaging standards of the two images to be merged are as close as possible, including slice thicknesses and imaging angulations. Today's stereotactic planning stations require some imaging standards to perform the best image fusion process with respect to their own image fusion algorithms. These detailed standards can be found each manufacturer's instructions for use (IFU) booklets. For example, no gantry 
tilted images are required for Medtronic Framelink planning station (Medtronic, Minneapolis, USA). In this sense, angulation difference of two different image series of a patient could be a fact that can fail the image fusion process. If the imaging procedures are performed in relation to a reference plane, this problem will be eliminated to a great extent. Most important planes used for frame fixation process are called Reid's baseline and the Glabella-Inion line ${ }^{14}$. Performing the frame fixation process with respect to these planes and having all stereotactic and non-stereotactic images with respect to these planes will avoid angulation differences. In our series we experienced image fusion failures in 85 procedures.

\section{CSF signal effect phenomenon on image fusion}

One of the most important radiological image series used in the planning phase of DBS surgery is the axial T1 MR images with contrast enhancement to visualize vascular structures ${ }^{15}$.The T2-weighed MR images are often used to directly visualize the target structures in DBS surgeries ${ }^{16}$.

Independent of the quality of the image fusion process performed for $\mathrm{T} 1$ and $\mathrm{T} 2$-weighed image series, the user may complain about the fusion quality of the images at the level of the lateral ventricles and the third ventricle. This perceived poor quality of fusion results from different signal characteristics of cerebrospinal fluid (CSF) in T2 and T1 images ${ }^{17}$. CSF containing structures appear to have different volumes due to different signal responses of CSF to different sequences (Figure 5A).

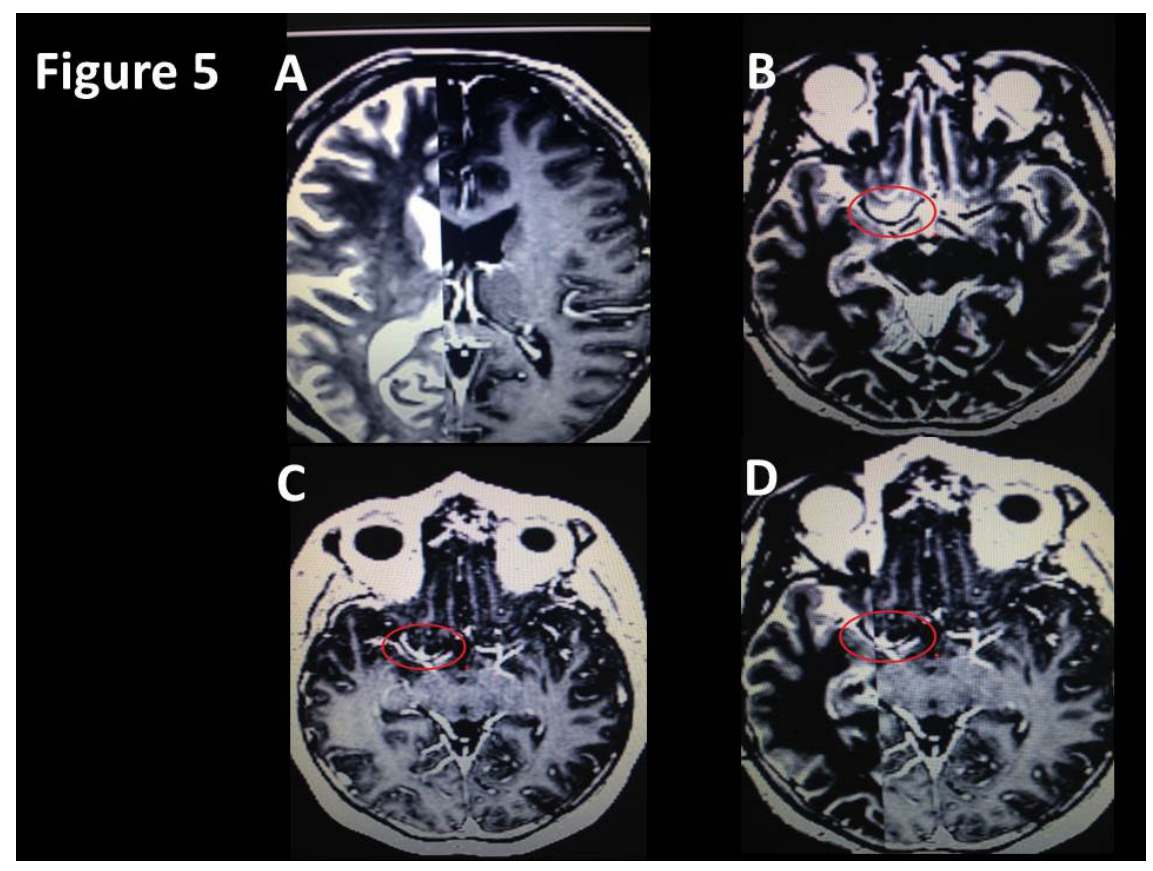

Figure 5:

Figure 5A shows $\mathrm{T} 1$ and $\mathrm{T} 2$ axial slices merged at the level of lateral ventricles. CSF containing structures have different volumes in $\mathrm{T} 1$ and $\mathrm{T} 2$ images. In figure $5 \mathrm{~B}$, the red circle shows the blood flow signal in a $\mathrm{T} 2$ axial slice. In figure $5 \mathrm{C}$, the red circle shows the same vascular structure with contrast agent in a $\mathrm{T} 1$ axial slice. In Figure 5D, the red circle shows the correlation between $\mathrm{T} 1$ and $\mathrm{T} 2$ images after fusion process.

Such volumetric discrepancies noticed in the manual fusion quality checks may cause the user to perceive as failed quality, even though the process has been completed successfully. At this point, we recommend using other anatomical structures instead of the ventricles to evaluate the quality of image fusion. Vascular structures, for instance, can be used for this purpose (Figure 5). The pericranium is also a useful structure to evaluate the accuracy of image fusion. In 25 of 921 procedures, these volumetric discrepancies were experienced.

\section{Discussion}

Here, we shared our experiences with stereotactic frame related complications. The first complication about stereotactic frame was described as movement related frame fixation problems. To solve this issue the best solution was detailed as using a stereotactic positioning aid device. The second complication topic about stereotactic frames was head anatomy related complications. For the patients with short neck, the sagittal anterior or sagittal posterior frame orientations and more inferior frame fixation methods were advised. Furthermore, oversized frontal sinus cavity related fixation problems were mentioned and the anatomical checks before fixation was advised. One another complication about stereotactic frame was detailed as lack 
of maintenance related complications. Performing the necessary checks after decontamination processes and regular maintenances after each usage has become a standard operation procedure in our centers.

In the second part, our experiences with stereotactic localizer related complications were described. Firstly, the compatibility of stereotactic localizer and planning station was underlined and the compatibility check was performed. Then, the missing fiducials on MR/CT scans due to the field of view effect was described. To avoid this, setting the FOV to an optimum scanning area which will not cause invisible fiducials on scans and which will not cause low resolution scans was implemented. Also, the problem of the presence of air bubbles in localizers using liquid solutions is mentioned to avoid a complication which may result as missing fiducial on radiological images. The last complication issue related to stereotactic localizers was imaging with disengaged or not fully engaged localizer. The junction points between the stereotactic frame and stereotactic localizer must be checked before the imaging process has been finalized.

In the third and the last part, planning station related complications were described. The first complication mentioned was the image fusion failures because of the imaging angulation differences between different series to be merged. Performing all the imaging with respect to one standard plane is advised for that issue. Secondly, the CSF signal on different MR series resulting unsatisfactory image fusion perception is described. Checking the image fusion quality through the vascular structures is advised as well. 


\section{References}

1. Kocabicak E, Terzi M, Alptekin O, Temel Y. Targeting thalamic tremor cells in deep brain stimulation for multiple sclerosis-induced complex tremor. Surgical Neurology International. 2013;4.

2. Kocabicak E, Temel Y, Höllig A, Falkenburger B, Tan SK. Current perspectives on deep brain stimulation for severe neurological and psychiatric disorders. Neuropsychiatric disease and treatment. 2015;11:1051.

3. Wichmann T, DeLong MR. Deep-brain stimulation for basal ganglia disorders. Basal ganglia. 2011;1(2):65-77.

4. Fenoy AJ, Simpson Jr RK. Risks of common complications in deep brain stimulation surgery: management and avoidance: Clinical article. Journal of neurosurgery. 2014;120(1):132-9.

5. Oh MY, Abosch A, Kim SH, Lang AE, Lozano AM. Long-term hardware-related complications of deep brain stimulation. Neurosurgery. 2002;50(6):1268-76.

6. Temel Y, Visser-Vandewalle V. Targets for deep brain stimulation in Parkinson's disease. Expert opinion on therapeutic targets. 2006;10(3):355-62.

7. Chan DT, Zhu XL, Yeung JH, et al. Complications of deep brain stimulation: a collective review. Asian J Surg. 2009;32(4):258-63.

8. Tan SK, Vlamings R, Lim L, et al. Experimental deep brain stimulation in animal models. Neurosurgery. 2010;67(4):1073-80.

9. Flusser J, Sroubek F, Zitova B. Image fusion: principles, methods, and applications. Tutorial Eusipco. 2007.

10. Dormont D, Ricciardi KG, Tandé D, et al. Is the subthalamic nucleus hypointense on T2-weighted images? A correlation study using MR imaging and stereotactic atlas data. American Journal of Neuroradiology. 2004;25(9):1516-23.

11. Plantinga BR, Temel Y, Roebroeck A, et al. Ultra-high field magnetic resonance imaging of the basal ganglia and related structures. Frontiers in human neuroscience. 2014;8.

12. Kocabıcak E AD, Ozaydın I, Jahanshahı A, K. H Tan S, Onar M, Boke O, Kurt M, Guz H, Terzı M, Alptekın O, Temel Y. Does Probe's Eye Subthalamic nucleus length on T2W MRI Correspond with Microelectrode Recording in Patients with Deep Brain Stimulation for Advanced Parkinson's Disease? Turk Neurosurg. 2013;23(5):658-65.

13. Hertel F, Husch A, Dooms G, Bernard F, Gemmar P. Susceptibility-Weighted MRI for Deep Brain Stimulation: Potentials in Trajectory Planning. Stereotactic and functional neurosurgery. 2015;93(5):303-8.

14. Tokunaga A, Takase M, Otani K. The glabella-inion line as a baseline for CT scanning of the brain. Neuroradiology. 1977;14(2):67-71.

15. Bériault S, Sadikot AF, Alsubaie F, Drouin S, Collins DL, Pike GB. Neuronavigation using susceptibility-weighted venography: application to deep brain stimulation and comparison with gadolinium contrast: Technical note. Journal of neurosurgery. 2014;121(1):131-41.

16. O'Gorman RL, Shmueli K, Ashkan K, et al. Optimal MRI methods for direct stereotactic targeting of the subthalamic nucleus and globus pallidus. European radiology. 2011;21(1):130-6.

17. Ortendahl DA, Posin JP, Hylton NM, Mills CM. Optimal visualization of cerebrospinal fluid on MRI. American journal of neuroradiology. 1986;7(3):403-7. 



\section{Chapter 3}

\section{Stereotactic accuracy and frame mounting: A phantom study}

Alptekin, O., Gubler, F. S., Ackermans, L., Kubben, P. L., Kuijf, M. L., Kocabicak, E., Temel, Y. (2019).

Stereotactic accuracy and frame mounting: A phantom study. Surgical Neurology International, 10. 


\begin{abstract}
Background: Frame mounting is considered one of the most critical steps in stereotactic neurosurgery. In routine clinical practice, the aim is to mount the frame as symmetrical as possible, parallel to Reid's line. However, sometimes, the frame is mounted asymmetrically often due to patient-related reasons.

Methods: In this study, we addressed the question whether an asymmetrically mounted frame influences the accuracy of stereotactic electrode implantation. A Citrullus lanatus was used for this study. After a magnetic resonance imaging scan, symmetric and asymmetric mounting of the frame, which could occur in clinical scenarios, was performed with computed tomography (CT). Three different stereotactic software packages were used to analyze the results. In addition, manual calculations were performed by two different observers.

Results: Our results show that an asymmetrically mounted frame (deviated, tilted, or rotated) does not affect the accuracy in the mediolateral axis (X-coordinate) or the anteroposterior axis (Y-coordinate). However, it can lead to a clinically relevant error in the superoinferior axis (Z-coordinate). This error was largest with manual calculations. These results suggest that asymmetrical frame mounting can lead to stereotactic inaccuracy in the superoinferior axis (Z-coordinate).
\end{abstract}

Conclusion: These results suggest that asymmetrical frame mounting can lead to stereotactic inaccuracy in the superoinferior axis ( $\mathrm{Z}$ coordinate). 


\section{Introduction}

Stereotactic neurosurgery is the technique for locating targets of surgical interest within the brain relative to an external frame of reference ${ }^{1}$. The application of stereotaxy has increased substantially with the introduction of deep brain stimulation (DBS) programs. Stereotaxy allows for reaching deeply located brain areas with high precision and minimal surgical exposure.

To reach a high level of precision in stereotactic surgeries, there are several sources of potential error which should be taken into account. Besides the mechanical errors of the stereotactic system itself, errors can occur due to imaging protocols and techniques and stereotactic planning software. An important step in stereotaxy is mounting the frame on the head of the patient. The aim is to mount the frame as symmetrical as possible parallel to Reid's line and/or Glabella-Inion line. However, an asymmetrically mounted frame is an often-seen condition, mostly due to patient-related reasons such as severe tremor, dystonia, or anxiousness. The question arises whether an asymmetrically mounted frame leads to stereotactic inaccuracy, and if yes, in which planes and to which extent? In this study, we addressed these questions. We have performed a phantom study using a Citrullus lanatus (watermelon). After mounting the frame in several potential clinical scenarios, magnetic resonance imaging (MRI) and stereotactical computed tomography (CT) were obtained. Subsequently, image fusion was performed using three commercially available stereotactic planning software systems. For comparison reasons, we also obtained manual calculations.

\section{Materials and Methods}

\section{Phantom}

We decided to use a phantom with natural properties, to obtain clear CT and MRI images. In this respect, we have chosen a C. lanatus (watermelon) with a form as close as possible to the human head. The rigid rind structure of a C. lanatus, with some reinforcement, was also sufficient to resist against the pressure that frame screws generate (Figure 1, Figure 2A). We implanted a human DBS (Medtronic 3389, Minneapolis, U.S.A.) electrode in the phantom. First, we performed an MRI scan. Then, we performed three stereotactic CT scans:

1. A scan with a symmetrically mounted frame (Figure 2B).

2. A scan with a rotational deviated mounting $-18^{\circ}$ rotation to the right side of the phantom (Figure $2 \mathrm{C}$ ).

3. A scan with a lateral tilted mounting $-10^{\circ}$ lateral tilt (Figure 2D).

The deviations and tilts were not too exaggerated to be able to mimic more or less a potential clinical situation. One specific segment, which was the deepest contact of the electrode (Medtronic 3389, Minneapolis) on the MRI images, was defined as the target for all three CT scans.

\section{Stereotactic frame}

A Leksell (Elekta, Stockholm, Sweden) Stereotactic G-frame was used during this study (Figure 1). The stereotactic arc was not used due to the design of the experiment. A N-localizer for the Leksell stereotactic frame was used to calculate the stereotactic coordinates of the target on both planning stations and manually (Figure 1). A universal frame adaptor was used as well, to fix the phantom and the frame to the table of the CT machine (Figure 1). We defined the anatomical surfaces as anterior, posterior, left, right, superior, and inferior. Just before mounting the frame for different scenarios, we attached some duct tapes to increase the resistance and the rigidity of the phantom's peel and to secure the fixation at the junction points between the phantom surface and the frame screws (Figure 1, Figure 2A). 


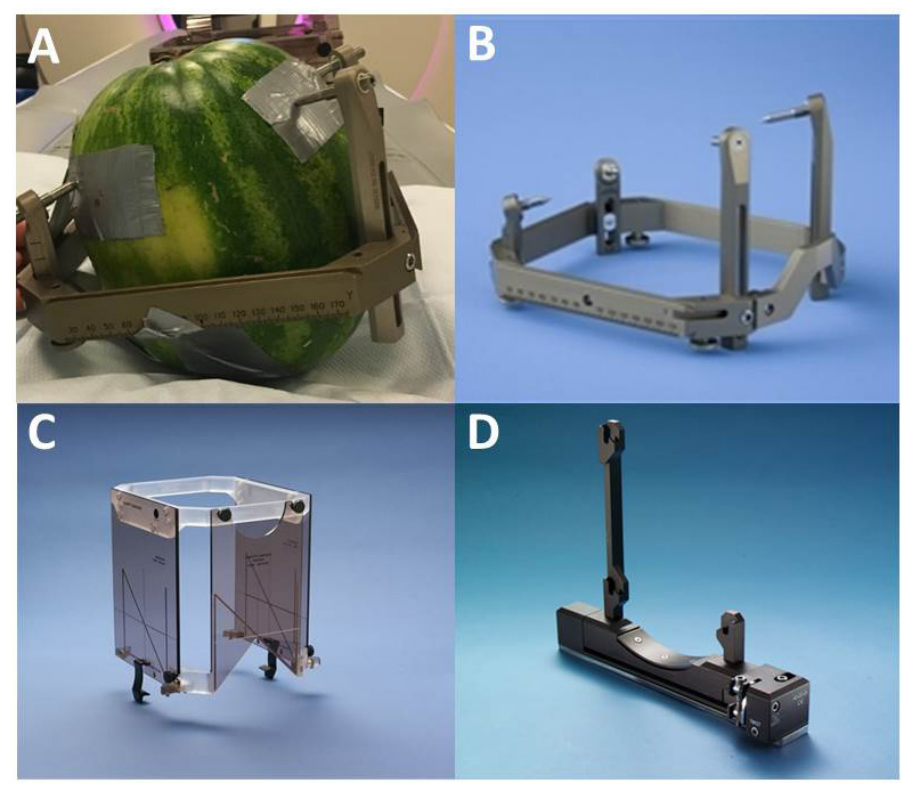

Figure 1: Figure 1A shows the phantom and the stereotactic frame mounted. In Figure $1 \mathrm{~B}$ the Leksell stereotactic frame is shown. The CT localizer used in the study is shown in Figure 1C and the universal CT adaptor in Figure 1D.

\section{DBS lead implantation}

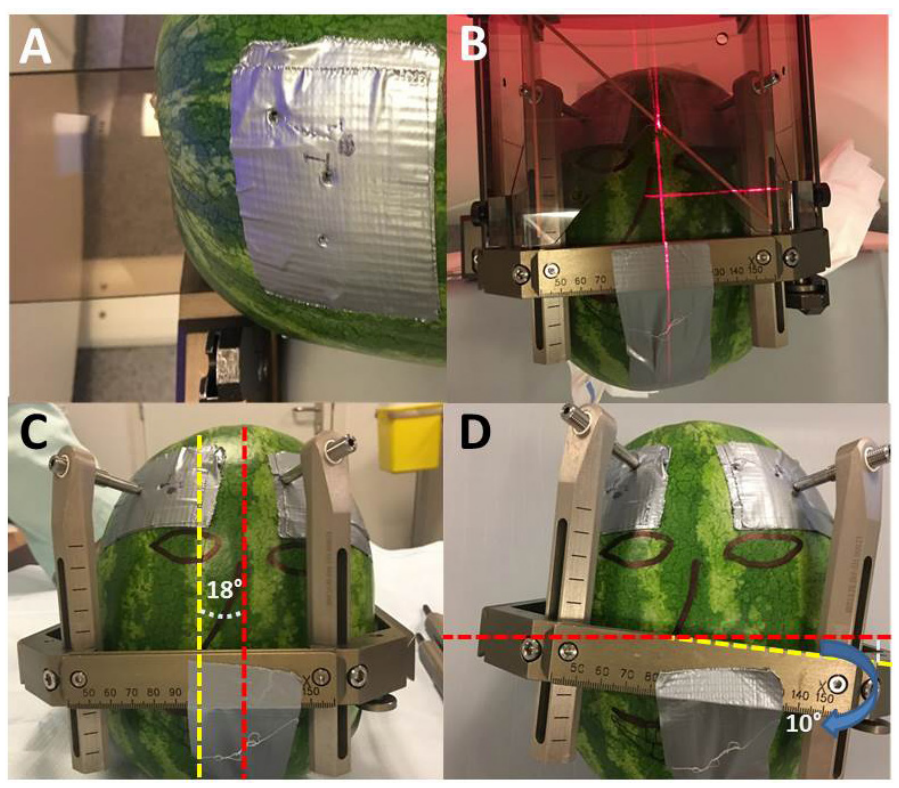

Figure 2: Figure 2A shows the duct tapes used to increase the resistance and the rigidity of the phantom's peel at the junction points between the phantom surface and the frame screws. In Figure $2 \mathrm{~B}$, the frame is mounted to the phantom symmetrically for scenario 1 . A scan with an $18^{\circ}$ rotational deviated mounting is shown in Figure 2C. The yellow dashed line is demonstrating the vertical midline of the frame and the red dashed line demonstrating the vertical midline of the phantom. The angle between two midlines related to the rotational deviation is also highlighted in Figure 2C. In Figure 2D, the stereotactic frame is mounted with a $10^{\circ}$ lateral tilt. The yellow dashed line is demonstrating the horizontal midline of the frame and the red dashed line demonstrating the horizontal midline of the phantom. The angle between two midlines related to the lateral tilt is also highlighted in Figure 2D.

A Medtronic 3389-28 (Medtronic, Minneapolis, USA) DBS lead was chosen for this study (Figure 3A). The lead was implanted after we made a trajectory for the electrode. We performed the burr hole with a micro drill (Dixi Medical, Besancon, France) and the tract was made with a FHC FC1019 (FHC, Bowdoin, USA) electrode guiding tube (Figure 3B). After the creation of the tract, the lead was descended through the tract manually until the tip of the electrode reached the end of this tract. The lead implantation was finalized after fixing the electrode with strong liquid glue. The glue dried and hardened, thus acting as a cement like fixation we normally use anchoring the electrode ${ }^{2}$. And the part of the electrode outside of the melon was cut short (Figure 3C).

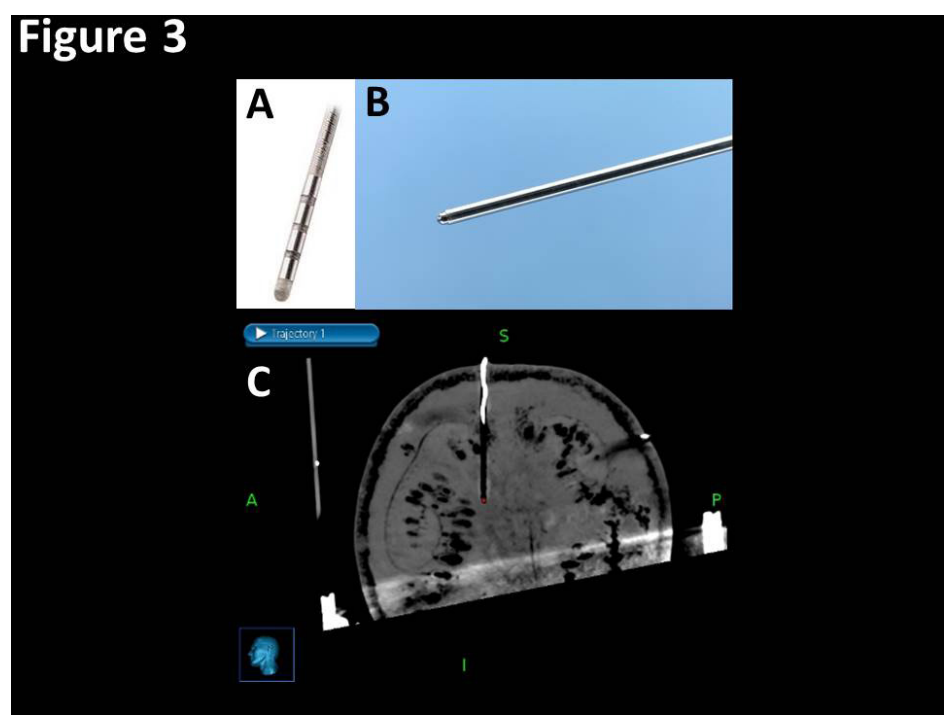

Figure 3:

Figure 3A shows the lead used in the study. In Figure 3B the cannula used for lead implantation is shown. In Figure $3 \mathrm{C}$ a sagittal reconstructed CT image of the phantom is shown. The implanted lead could also be seen in the same image. 
The target was defined as the deepest contact of the DBS electrode as seen on the MRI. The center of the circle with black contrast which is the artifact of DBS electrode was chosen as the target for all three scenarios (Figure 4).

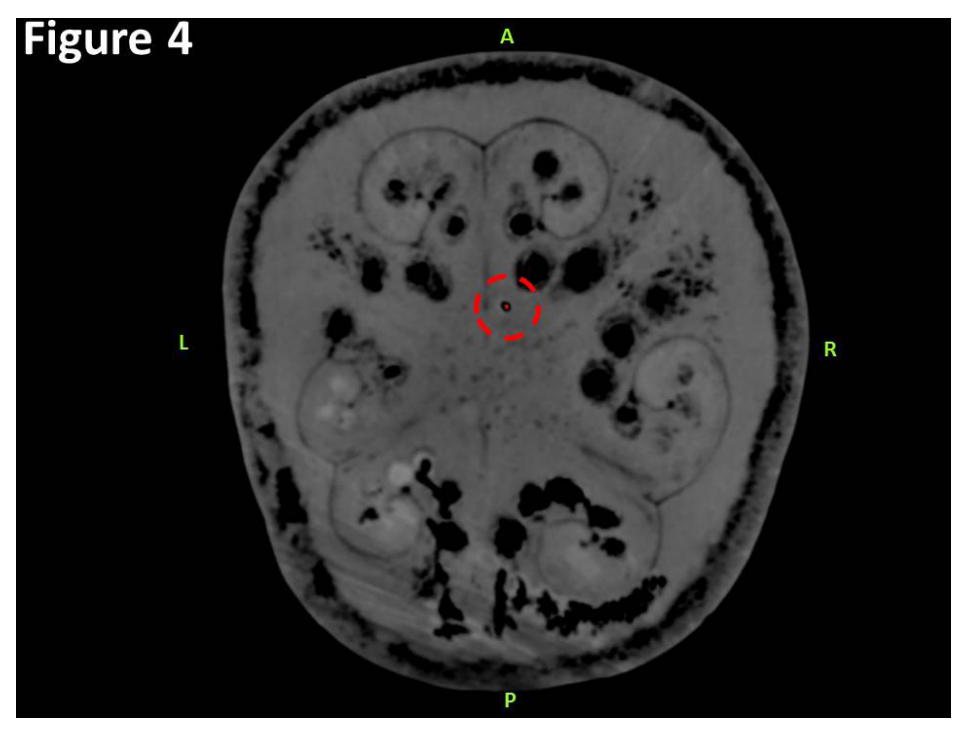

Figure 4:

The second deepest CT slice on which that DBS electrode was clearly visible is shown in Figure 4. The red dot which is highlighted with dashed circle is the target point for all 3 scenarios.

\section{Imaging and image processing}

We performed a T2-weighted MRI scan to get nonstereotactic MR images of the lead implanted phantom. A 1.5 Tesla (T), Philips Ingenia (Philips, Eindhoven, the Netherlands) MRI machine was used in this phase. Slice thickness for T2-weighted MR images was $2 \mathrm{~mm}$ with no angulation. After mounting the frame, each time we performed a stereotactic CT scan for the three different frame-mounted scenarios (Figure 5A-C). A Siemens Somatom Force (Siemens, Munich, Germany) CT machine was used to obtain the stereotactic CT images. No angulation was applied, and slice thicknesses for each scan were $0.6 \mathrm{~mm}$.

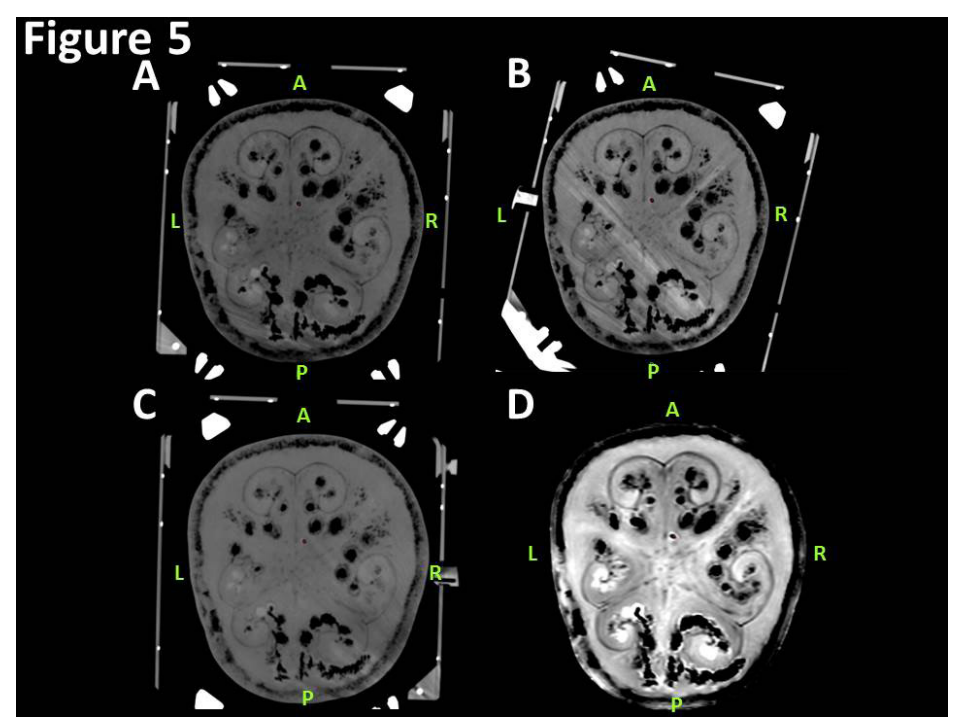

Figure 5:

In Figure 5, stereotactic CT slices for 3 scenarios (Figure $5 \mathrm{~A}$ for Scenario 1, Figure 5B for Scenario 2, Figure 5C for Scenario 3 ) at the target point and the T2 weighted axial MRI scan slice at the same target point (Figure 5D) is shown.

The targeting phases were performed both computer-based and manually. For the computer-based analyses, we used three different planning stations:

1. Medtronic Framelink version 5.4.1 (Medtronic, Minneapolis, USA),

2. Medtronic Cranial Software (Medtronic, Minneapolis, USA),

3. Brainlab iPlan (Brainlab, Feldkirchen, Germany).

Since we had three different stereotactic CT scans for three different scenarios, MRI T2 series were chosen as registration series and all three $\mathrm{CT}$ series were merged with $\mathrm{T} 2$ axial images (Figure 5D). Image fusion was processed with the automatic algorithm of the software and the results were checked for each merged series. Auto-merge algorithms of planning stations were successful for all three merged image groups. 
For each mounting scenario, we registered the related stereotactic CT series to Framelink 5.4.1, Medtronic Cranial and Brainlab iPlan software. The stereotactic coordinates generated for the target point by three planning stations for each three frame mounting scenario were recorded. The targeting was also performed manually using the method which is defined for N-Localizers ${ }^{3}$. The outputs for the manual calculations were obtained using the measuring tool of Medtronic Cranial Software (Figure 6) and recorded like the previous outputs.

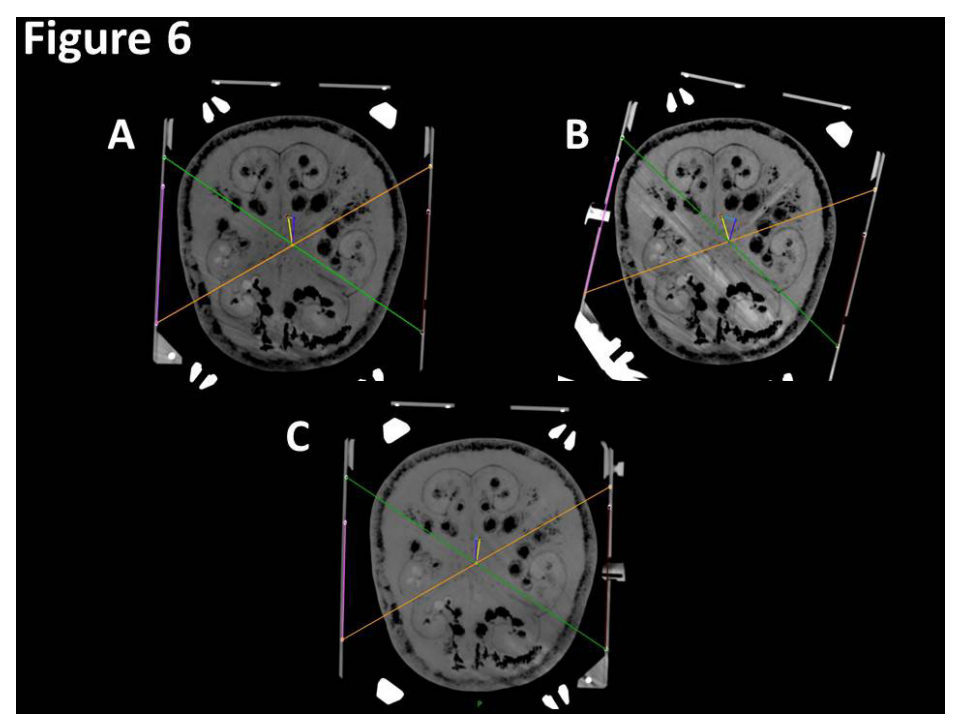

Figure 6:

In Figure 6, the manual targeting performed in Medtronic Cranial software for three mounting scenarios (Figure 6A for Scenario 1, Figure 6B for Scenario 2, Figure 6C for Scenario 3 ) at the target point for N- Localizers are shown.

Manual calculations were done twice separately by the two different observers (Table 1). The measurements obtained by two observers' manual calculations were analyzed with IBM SPSS Statistics 25 . The inter- and intraobserver reliabilities were measured using Kendall's coefficient of concordance (W) and were recorded (Table 2).

\begin{tabular}{|c|c|c|c|}
\hline & $X(\mathrm{~mm})$ & $Y(\mathrm{~mm})$ & $Z(\mathrm{~mm})$ \\
\hline Medtronic Framelink 5.4.1 & 103.8 & 119.6 & 136.7 \\
\hline Medtronic Cranial & 104.4 & 119.6 & 135.8 \\
\hline Brainlab & 104.6 & 119.5 & 136.1 \\
\hline Manual Calculation Observer 1.0 & 104.2 & 119.7 & 134.24 \\
\hline Manual Calculation Observer 1.1 & 104.4 & 120.2 & 135.6 \\
\hline Manual Calculation Observer 2.0 & 104.3 & 119.5 & 135.5 \\
\hline Manual Calculation Observer 2.1 & 103.9 & 119.9 & 135.65 \\
\hline$\Delta$ & 0.8 & 0.7 & 2.46 \\
\hline \multicolumn{4}{|c|}{ Scenario 2 - A scan with a rotational deviation } \\
\hline & $X(\mathrm{~mm})$ & $Y(\mathrm{~mm})$ & $Z(\mathrm{~mm})$ \\
\hline Medtronic Framelink 5.4.1 & 97.4 & 117.7 & 139.1 \\
\hline Medtronic Cranial & 97.5 & 118.2 & 138.5 \\
\hline Brainlab & 97.7 & 118 & 139.4 \\
\hline Manual Calculation Observer 1.0 & 97.2 & 118 & 136.4 \\
\hline Manual Calculation Observer 1.1 & 97.4 & 118.3 & 137.2 \\
\hline Manual Calculation Observer 2.0 & 97.5 & 118.2 & 137.3 \\
\hline Manual Calculation Observer 2.1 & 97.6 & 117.9 & 137.3 \\
\hline$\Delta$ & 0.5 & 0.6 & 3 \\
\hline \multicolumn{4}{|c|}{ Scenario 3 - A scan with a medio-lateral deviation } \\
\hline & $X(\mathrm{~mm})$ & $Y(\mathrm{~mm})$ & $Z(\mathrm{~mm})$ \\
\hline Medtronic Framelink 5.4.1 & 109.9 & 117.5 & 139.6 \\
\hline Medtronic Cranial & 110.1 & 117.7 & 138.9 \\
\hline Brainlab & 110.6 & 117.4 & 138.9 \\
\hline Manual Calculation Observer 1.0 & 110,7 & 117 & 135.8 \\
\hline Manual Calculation Observer 1.1 & 110.3 & 117.4 & 136.55 \\
\hline Manual Calculation Observer 2.0 & 110.4 & 117.6 & 137.45 \\
\hline Manual Calculation Observer 2.1 & 110.9 & 117.3 & 137.25 \\
\hline$\Delta$ & 1 & 0.7 & 3.8 \\
\hline
\end{tabular}
Table 1:

$\mathrm{X}, \mathrm{Y}$ and $\mathrm{Z}$ coordinates obtained by using 3 different planning softwares and by using manual calculation method for the same target point calculated by two different observers for three different scenarios are listed in Table 1. Calculations named as Observer 1.0 and 1.1 belongs to the Observer 1 and calculations named as Observer 2.0 and 2.1 belongs to the Observer 2. The differences between the highest measurements and the lowest measurements for each coordinate are defined as delta $(\Delta)$. Each scenario has its own $\Delta$ calculations for obtained $\mathrm{X}$, $\mathrm{Y}$ and $\mathrm{Z}$ coordinates. 


\begin{tabular}{|c|c|c|c|c|c|c|}
\hline \multicolumn{7}{|c|}{ Correlations } \\
\hline & & & Observer1.0 & Observer1.1 & Observer 2.0 & Observer 2.1 \\
\hline \multirow[t]{12}{*}{ Kendall's tau_b } & \multirow[t]{3}{*}{ Observer1.0 } & Correlation Coefficient & 1.000 & $1.000^{* \pi}$ & $.944^{\prime \prime}$ & $1.000^{* \prime}$ \\
\hline & & Sig. (2-tailed) & . & . & .000 & . \\
\hline & & $N$ & 9 & 9 & 9 & 9 \\
\hline & \multirow[t]{3}{*}{ Observer1.1 } & Correlation Coefficient & $1.000^{* *}$ & 1.000 & $.944^{* \prime}$ & $1.000^{* \prime}$ \\
\hline & & Sig. (2-tailed) & . & . & .000 & . \\
\hline & & $N$ & 9 & 9 & 9 & 9 \\
\hline & \multirow[t]{3}{*}{ Observer 2.0} & Correlation Coefficient & $.944^{\prime \prime}$ & $.944^{*}$ & 1.000 & $.944^{* \prime}$ \\
\hline & & Sig. (2-tailed) & .000 & .000 & . & .000 \\
\hline & & $N$ & 9 & 9 & 9 & 9 \\
\hline & \multirow[t]{3}{*}{ Observer 2.1} & Correlation Coefficient & $1.000^{* *}$ & $1.000^{* *}$ & $.944^{* \prime}$ & 1.000 \\
\hline & & Sig. (2-tailed) & . & . & .000 & . \\
\hline & & $N$ & 9 & 9 & 9 & 9 \\
\hline
\end{tabular}

Table 2: The inter-observer and intra-observer reliabilities measured by using Kendall's Coefficient of Concordance (W) are presented in Table 2. Table 2 is the output of the observers' measurements processed by IBM SPSS Statistics 25 software.

\section{Results}

For the rotational deviation scenario, the deviation angle was measured as $18^{\circ}$ (Figure $2 \mathrm{C}$ ). The angle measured in lateral deviation scenario was $10^{\circ}$ (Figure 2D).

\section{Scenario 1 - A scan with a symmetrically mounted frame}

For scenario 1, the absolute difference between the highest (Brainlab iPlan-104.6 mm) and the lowest (Framelink 5.4.1 mm-103.8 mm) measurements for X-coordinate was $0.8 \mathrm{~mm}$. The absolute difference between the highest (Manual calculation - $120.2 \mathrm{~mm}$ ) and the lowest (Brainlab Iplan - $119.5 \mathrm{~mm}$ ) measurements for Y-coordinate in scenario 1 was $0.7 \mathrm{~mm}$. For the Z-coordinate, the absolute difference between the highest (Framelink 5.4.1 $\mathrm{mm}-136.7 \mathrm{~mm}$ ) and the lowest (Manual calculation - $134.24 \mathrm{~mm}$ ) measurements in scenario 1 was $2.46 \mathrm{~mm}$.

\section{Scenario $2-A$ scan with a rotational deviated mounting}

For scenario 2, the absolute difference between the highest (Brainlab Iplan - $97.7 \mathrm{~mm}$ ) and the lowest (Manual clculation - $97.2 \mathrm{~mm}$ ) measurements for X-coordinate was $0.5 \mathrm{~mm}$. The absolute difference between the highest (Manual calculation $-118.3 \mathrm{~mm}$ ) and the lowest (Framelink $5.4 .1 \mathrm{~mm}-117.7 \mathrm{~mm}$ ) measurements for Y-coordinate in scenario 2 was $0.6 \mathrm{~mm}$. For the Z-coordinate, the absolute difference between the highest (Brainlab iPlan $139.4 \mathrm{~mm}$ ) and the lowest (Manual calculation - $136.4 \mathrm{~mm}$ ) measurements in scenario 2 was $3 \mathrm{~mm}$.

\section{Scenario 3 - A scan with a lateral tilted mounting}

For scenario 3, the absolute difference between the highest (Manual calculation $-110.9 \mathrm{~mm}$ ) and the lowest (Framelink 5.4.1 mm-109.9 mm) measurements for X-coordinate was $1.0 \mathrm{~mm}$. The absolute difference between the highest (Medtronic Cranial - $117.7 \mathrm{~mm}$ ) and the lowest (Manual calculation - $117 \mathrm{~mm}$ ) measurements for Y-coordinate in scenario 3 was $0.7 \mathrm{~mm}$. For the Z-coordinate, the absolute difference between the highest (Framelink 5.4.1 mm-139.6 mm) and the lowest (Manual calculation - $135.8 \mathrm{~mm}$ ) measurements scenario 3 was $3.8 \mathrm{~mm}$. 
For the values obtained through manual calculations from all three scenarios, we calculated the intra- and interobserver reliability using Kendall's Coefficient of Concordance (W). All the W values in between observers and their observations were above 0.94 (Table 2).

\section{Discussion}

Here, we addressed the question whether a clinically relevant asymmetrical mounting of the frame will result in stereotactic inaccuracy and found that a rotationally deviated frame mounting and laterally tilted frame mounting will not affect the accuracy in the mediolateral axis/X-coordinate or anteroposterior axis/Ycoordinate but can lead to an inaccuracy in the superoinferior axis/Z-coordinate.

For the mediolateral axis/X-coordinate, the mean absolute error in this study was $0.76 \mathrm{~mm}$ (standard deviation [SD]: \pm 0.14$)$. An earlier study found that the mean error of targeting using frame-based systems in the mediolateral axis/X-coordinate was $1.0 \mathrm{~mm}(\mathrm{SD} \pm 0.7)^{4}$. The mean absolute errors calculated for the three mounting experiments for the X-coordinate are below $1 \mathrm{~mm}$ and therefore seem to be not clinically relevant. The situation for the anteroposterior axis/Y-coordinate is similar. The absolute mean error calculated in this study was $0.66 \mathrm{~mm}(\mathrm{SD}: \pm 0.21)$. This error has been found to be $0.9 \mathrm{~mm}(\mathrm{SD} \pm 0.5)$ in the abovementioned study ${ }^{4}$. However, for the superoinferior axis/Z-coordinate, the absolute mean error in this study was $3.09 \mathrm{~mm}$ (SD: \pm 0.55 ), which is a substantial deviation. In two other studies, the general stereotactic error in the Z-coordinate was $0.7 \mathrm{~mm}(\mathrm{SD} \pm 0.6)(6)$ and $1.3 \mathrm{~mm}(\mathrm{SD} \pm 0.6)^{5}$. If we divide the results in software- and manual-calculated parameters, then the mean absolute error of the Z-coordinates obtained from the software is actually within the range reported in the literature $(>1.3 \mathrm{~mm} \mathrm{SD} \pm 0.6)$. Due to the contribution of manual calculation technique to the mean absolute error calculations for all three data sets, the mean absolute error results appear higher than the values reported in literature. With respect to the results of our experiment and reported errors in literature, we suggest that manual calculations can lead to an inaccuracy of $>1.3 \mathrm{~mm}$ in the superoinferior axis/Z-coordinate. Moreover, in the scenario of a deviated mounted frame, these inaccuracies increase.

The accuracy of stereotactic techniques and systems has been investigated for a long time. One study has questioned geometric accuracy of three-dimensional (3D) coordinates of the Leksell stereotactic frame in 1.5 T and 3.0 T MRI with different fixation screw materials ${ }^{6}$. They concluded that the geometric accuracy of the Leksell skull frame system with 1.5 T MR imaging was high and valid for clinical use. However, the geometric errors with 3.0 T MR imaging were larger than those of 1.5 T MR imaging and were acceptable only with aluminum cranial quick fixation screws. Another study has investigated their targeting accuracy with three different techniques ${ }^{7}$. The first technique involved determination of anatomical landmarks and fiducial markers of the stereotactic frame on the monitor screen of an MRI scanner and calculation of the target point using a series of formulas; the second technique used a Leksell tabletop localizer, and the third technique used a stereotactic navigation software. They concluded that the use of computerized planning software increased the precision of target coordinate calculation and improves the accuracy of functional stereotactic procedures, thus strengthening our findings.

Nowadays, new technologies are still being developed by manufacturers to achieve the perfect accuracy in another stereotactic technique which is called frameless technique. The accuracy comparison between framebased and frameless techniques had also been questioned and published ${ }^{5}$. In one study, the targeting accuracy of Nextframe (Medtronic, Minneapolis, USA) frameless stereotactic system and Leksell frame-based stereotactic system has been compared. They concluded that both techniques have equivalent overall 3D accuracy. These comparisons and studies are beyond the scope of this article. As far as we know, there are no specific studies questioning the stereotactic accuracy through a frame mounting perspective. 
For the superoinferior/Z-axis coordinates, the highest inaccuracy for all three experiments is from the manual calculation technique. Since the manual calculation method is an observer-based targeting method, we obtained the manual-calculated coordinates from two independent observers and these two observers performed their manual calculations twice for each scenario. The intra- and inter-observer reliabilities we achieved were very high which led us to confirm that the source of deviation achieved from the superoinferior/Z-axis is not related to observer measurements. In another study, it was advised to ensure that the axes of the frame are in line with those of the scanner when manual calculation methods are considered for targeting. According to the same study, with this particular attention, frame geometry is reproduced accurately on a cross-sectional imaging ${ }^{8}$.

In this study, all CT images were obtained with respect to the alignment of the scanner axis and frame axes. Since the reference or registration image series were T2-weighted nonstereotactic series, the alignment of the CT scan could have been changed after the image fusion process. The manual targeting calculations in this study were performed on these merged stereotactic images using the measurement tools of Medtronic Cranial (Medtronic, Minneapolis, USA) software. We also believe that this difference could also arise with respect to the sensitivity difference between computer-based algorithms designed for auto-detection of fiducials and the direct/manual registration performed by the user.

When we compare the clinical applications of stereotactic planning software in DBS procedures and the design of this study, another difference arises through registration series perspective. In clinical applications of stereotactic planning software, CT series are commonly used as registration series ${ }^{9}$. As detailed in this study's design, MRI T2 series were chosen as registration series. Due to this difference, we investigated if the different registration series (MRI) might affect the stereotactic accurcy, specific the value of the Z-coordinate, in our study. The choice of registration series and its reflection to stereotactic accuracy has been studied in another study, and no significant difference was found between CT and MRI series ${ }^{10}$. However, in our experimental design, we used a non-stereotactic MRI as registration series and fused it with a stereotactic CT. A possible explanation for the inaccuracy in the Z-coordinate could be due the stereotactic frame deviation after the anatomical image fusion by the software.

In the clinical applications of the stereotactic software, the systems require some anatomical important reference points to be registered from the radiological images manually by the user. These reference points are required for the definition of anterior commissure (AC), posterior commissure (PC), and the mid-sagittal plane (MSP) ${ }^{11}$. From DBS procedures perspective, these reference points are mainly used when the indirect targeting method is considered ${ }^{12}$. Second, these reference points will give the user preplanning option before the frame is mounted to the patient head. At this point, another question appears whether registration of these reference points will affect the accuracy comparison between three mounting scenarios. Then, registration of some structures which could easily been identified or seen as AC, PC, and MSP in the phantom was discussed. In this study, the target coordinates were calculated with respect to the fiducial markers registered to the software. Furthermore, we performed direct targeting method for the aimed part of the implanted DBS lead. Due to the technical aspects of the stereotactic planning software and the targeting method performed, we agreed that registration of these reference points will not affect the accuracy comparison of these three mounting scenarios.

\section{Conclusion}

In stereotactic neurosurgical procedures, the superoinferior axis (Z-coordinate) is susceptible for inaccuracy when manual calculations are applied and increase with an asymmetrical-mounted stereotactic frame. Furthermore, our findings suggest that using a stereotactic scan as the registration series can reduce inaccuracies when compared to nonstereotactic scans. 


\section{References}

1. Maciunas RJ, Galloway Jr RL, Latimer JW. The application accuracy of stereotactic frames. Neurosurgery. 1994;35(4):682-95.

2. Smeets AY, Ackermans L, Oosterloo M, et al. Modified cement-based fixation of the deep brain stimulation electrode. Stereotactic and functional neurosurgery. 2015;93(1):67-.

3. Sharan AD, Andrews DW. Stereotactic frames: technical considerations. NEUROLOGICAL DISEASE AND THERAPY. 2003;58:11-20.

4. Sharma M, Rhiew R, Deogaonkar M, Rezai A, Boulis N. Accuracy and precision of targeting using frameless stereotactic system in deep brain stimulator implantation surgery. Neurology India. 2014;62(5):503.

5. Bot M, Van Den Munckhof P, Bakay R, Sierens D, Stebbins G, Metman LV. Analysis of stereotactic accuracy in patients undergoing deep brain stimulation using Nexframe and the Leksell frame. Stereotactic and functional neurosurgery. 2015;93(5):316-25.

6. Nakazawa H, Mori Y, Yamamuro O, et al. Geometric accuracy of 3D coordinates of the Leksell stereotactic skull frame in 1.5 Tesla-and 3.0 Tesla-magnetic resonance imaging: a comparison of three different fixation screw materials. Journal of radiation research. 2014;55(6):1184-91.

7. Slavin KV, Anderson GJ, Burchiel KJ. Comparison of three techniques for calculation of target coordinates in functional stereotactic procedures. Stereotactic and functional neurosurgery. 1999;72(2-4):192-5.

8. Zrinzo L. Pitfalls in precision stereotactic surgery. Surgical neurology international. 2012;3(Suppl 1):S53.

9. Mirzadeh Z, Chapple K, Lambert M, Dhall R, Ponce FA. Validation of CT-MRI fusion for intraoperative assessment of stereotactic accuracy in DBS surgery. Movement Disorders. 2014;29(14):1788-95.

10. Sharma M, Deogaonkar M. Accuracy and safety of targeting using intraoperative "O-arm" during placement of deep brain stimulation electrodes without electrophysiological recordings. Journal of Clinical Neuroscience. 2016;27:80-6.

11. Schaltenbrand G. Atlas for stereotaxy of the human brain. Georg Thieme. 1977.

12. Rabie A, Verhagen Metman L, Slavin KV. Using "functional” target coordinates of the subthalamic nucleus to assess the indirect and direct methods of the preoperative planning: do the anatomical and functional targets coincide? Brain sciences. 2016;6(4):65. 


\section{Chapter 4}

Impact of Computed Tomography Image Slice Thickness on Stereotaxy:

\section{A Phantom Study}

Alptekin, O., Gubler, F. S., Ackermans L., Kubben P.L., Kuijf, M.L., Kocabicak, E., Temel, Y. 


\section{Abstract}

Background: Magnetic Resonance imaging (MRI) and stereotactic Computed Tomography(CT) co-registration is often applied for stereotactic deep brain stimulation (DBS) surgeries in order to combine multiple sources of information. The slice thickness of a (stereotactic) CT is a matter of discussion but often thin slices are used and recommended because of the high reported accuracy. In this study we investigate if the stereotactic CT slice thickness has an effect on stereotactic calculations and its accuracy.

Methods: We used a phantom, a citrullus lanatus (watermelon), implanted with a DBS electrode. The deepest contact point of the electrode was the defined fixed target. Coordinates and ring and arc angles from all stereotactic CT scans with different slice thicknesses of $0.5 \mathrm{~mm}, 1.0 \mathrm{~mm}, 1.5 \mathrm{~mm}, 2.0 \mathrm{~mm}, 3.00 \mathrm{~mm}, 4.00 \mathrm{~mm}$ and $5 \mathrm{~mm}$ were recorded and compared after fusion with a thin sliced non-stereotactic MRI.

Results: Our results show no significant differences in stereotactic calculations for the different CT's with all absolute mean differences in millimeter $(\mathrm{mm})$ for the stereotactic coordinates $<0.5$ and angles in degrees $<$ 0.4 .

Conclusion: We found no significant effect of increasing slice thickness of CT on the stereotactic calculation when fused with a thin-sliced MRI in a simulated stereotactic DBS setting. 


\section{Introduction}

Deep brain stimulation (DBS) is a well-known stereotactic application of neuromodulation. Stereotaxy with DBS allows the neurosurgeon to reach and modulate deep brain structures in a minimal invasive way ${ }^{1}$.

Stereotactic surgery technique relies on identification of the desired targets in the brain and calculation of their position with respect to an extracranial coordinate system (X, Y, Z). The calculations are performed based on the registration of the stereotactic frame with the patient's anatomy and the analysis of radiological images ${ }^{2}$. To obtain these desired coordinates, there are two methodologies. In the early days, manual calculations were performed $^{3,4}$. Nowadays, with the development of computerized technologies, the targeting and calculations have become automatic, user friendly, more precise, faster and more reliable than the manual option ${ }^{5,6}$.

The use of (contrast-enhanced) Magnetic Resonance Imaging (MRI) in the planning and targeting of these procedures, due to its superior capability to visualize the anatomical structures and correct identification of cerebral vasculature, is widely accepted. However, stereotactic information can be extracted from both MRI and computed tomography (CT) images due to the configuration of the stereotactic equipment. In an equivalent, several experienced centers recommending the use of CT to obtain stereotactic information, rather than a stereotactic MRI. The main disadvantage of MRI is the geometric distortion related to the presence of the stereotactic frame during the scan in comparison with $\mathrm{CT}^{7-9}$. Furthermore, practical considerations in favor of CT are patient comfort, scanning time and patient safety. Transportation of (sedated and intubated) patients for stereotactic imaging is often necessary because mostly scanners are not available on the operation room (OR). Therefore, the quicker the scanning is performed the better. Lastly, regarding cranial applications CT scans are more cost effective in comparison to MR scans ${ }^{10,11}$. The co-registration of a stereotactical CT with a MRI brings together the best of both worlds.

In conclusion, the use of CT for stereotaxy is easy, patient friendly, safe and cost effective. However, the slice thickness of a stereotactic CT is a matter of discussion. Often thin slices $(\sim 1 \mathrm{~mm})$ are used and/or recommended because of high reported accuracy ${ }^{12-14}$. There is very limited information in the literature on the slice thickness of the CT scans ${ }^{15,16}$. Moreover, and as far as we are aware of, the effect of stereotactic CT slice thickness on stereotactic calculations has not been reported.

In this study, we addressed the question whether slice thickness of stereotactic CT images influences computerbased calculations, and thus its stereotactic accuracy, in a simulated stereotactic DBS setting, and if so, to what extent.

\section{Materials and Methods}

\section{Phantom}

A citrullus lanatus (watermelon) was used as phantom to get clear MR and CT images. As showed in our former study, its form is close to a human head and its biological consistent well suited for both image modalities simulating a real patient. Therefore, mounting a stereotactic frame and implanting an electrode is very convenient ${ }^{6}$. We implanted a human DBS electrode (Medtronic 3389, Minneapolis, U.S.A.) in the phantom. First, we performed a non-stereotactic T2 MRI scan, i.e. without a stereotactical frame, with the implanted phantom. Then, seven stereotactic CT scans were made with $0.5 \mathrm{~mm}, 1 \mathrm{~mm}, 1.5 \mathrm{~mm}, 2 \mathrm{~mm}, 3 \mathrm{~mm}$, $4 \mathrm{~mm}$ and $5 \mathrm{~mm}$ slice thicknesses, respectively. These slice thickness values were chosen based upon regular clinical usage ranges and slice thickness ranges used in three-dimensional (3D) reconstruction studies ${ }^{17}$. 
A Leksell (Elekta, Stockholm, Sweden) Stereotactic G-frame was used. A N-Localizer for the Leksell stereotactic frame was used to calculate the stereotactic coordinates of the target on the planning station. A universal frame adaptor was used, to fix the phantom and the frame to the CT scan table.

\section{DBS lead implantation}

A Medtronic 3389-28 (Medtronic, Minneapolis, USA) DBS lead was used for this study. We made a trajectory in the phantom and implanted the lead manually through the created path until the tip of the electrode reached the end of the trajectory (Figure1A). The lead was fixated to the phantom with a strong liquid glue (Pattex $100 \%$, Henkel, Düsseldorf, Germany). The part of the electrode remaining outside of the phantom was cut after the glue was hardened.

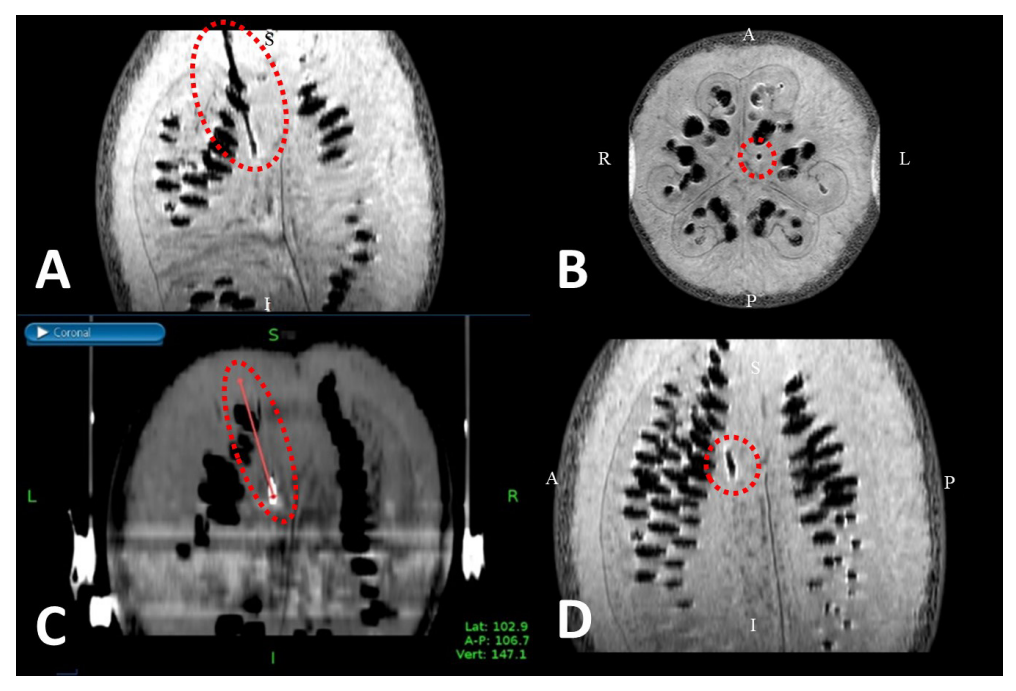

Figure 1:

In Figure $1 \mathrm{~A}$, a cross-sectional T2 MR image of the phantom and the lead is shown. The orientation of the image is reconstructed in order to visualize the lead and its tract. The fourth axial image from T2 series where the electrode has its first largest volume is shown in Figure 1 $\mathrm{B}$. The electrode is highlighted with the red-dashed circle. In Figure $1 \mathrm{C}$, the drawn trajectory with the planning station is highlighted with red-dashed elliptic circle. A sagittal reconstructed image from T2 MR images of the lead and phantom is shown. The lead is highlighted with the red-dashed circle.

\section{Imaging, Image Processing and Targeting}

We performed a T2 weighted MRI scan to get non-stereotactic MR images of the lead implanted phantom. A 1.5T, Philips Ingenia (Philips, Eindhoven, the Netherlands) MRI machine was used (Figure 2A). The phantom was fixated on the MRI table in the head holder with the head coil similar to a patient's head using pillows. Imaging parameters for T2 MRI were TE: 100, TR: 4941 and the voxel size was $0.76 \mathrm{~mm}, 0.99 \mathrm{~mm}$ and $2.00 \mathrm{~mm}$.
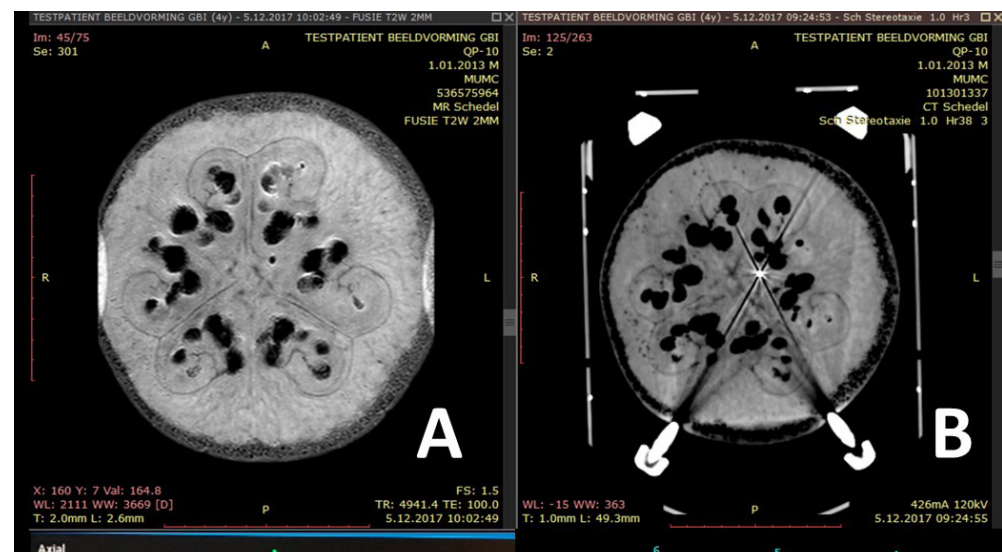

Figure 2:

In figure $2 \mathrm{~A}$, an axial slice from $\mathrm{T} 2$ weighted MR images of the phantom and the predefined target point is shown. A stereotactic CT slice of the same phantom is shown in Figure 2B. In Figure 2C, co-registration of MR and CT images of the phantom is shown. The auto registration of the N-localizer from the axial stereotactic CT slice is shown in Figure 2D.

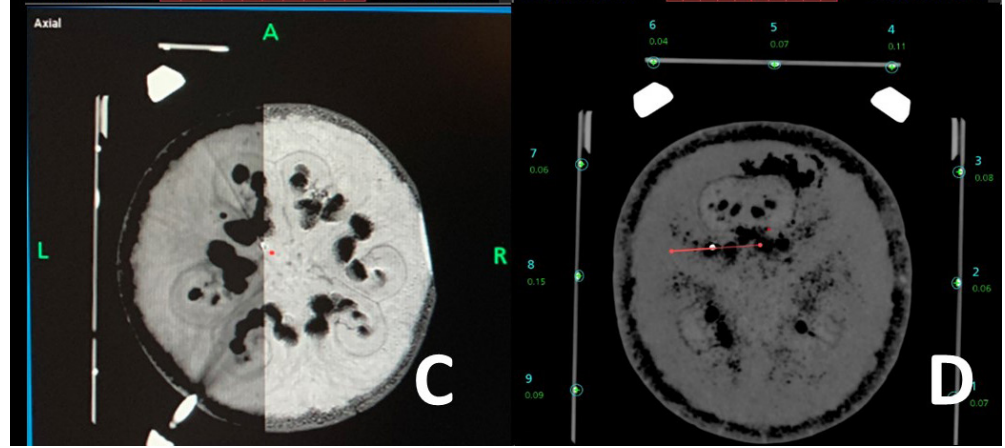


Directly after the MRI, in order to avoid possible structural changes of the phantom like putrefy, the stereotactic frame was mounted as previously described and we performed seven stereotactic CT scans using a Siemens Somatom Force (Siemens, Munich, Germany) helical image acquisition CT system (Figure 2B) ${ }^{6}$. Field of view, milliampere-second and kilovoltage parameters for all CT series were $276 \mathrm{~mm}, 638 \mathrm{mAs}$ and $120 \mathrm{kV}$ respectively. No gantry angulation was applied and we obtained seven different series with $0.5 \mathrm{~mm}, 1 \mathrm{~mm}$, $1.5 \mathrm{~mm}, 2 \mathrm{~mm}, 3 \mathrm{~mm}, 4 \mathrm{~mm}$ and $5 \mathrm{~mm}$ slice thicknesses using the image processing tools within the CT machine.

The targeting procedure was performed with a Medtronic S7 Workstation/ Cranial Software (Medtronic, Minneapolis, USA). The deepest contact point of the electrode was the defined fixed target. The axial image from the T2 series where the deepest contact has the largest volume was defined as target slice (Figure 1B). The center of the circle with black contrast which is the artifact of DBS electrode was chosen as the fixed point. Additionally, in order to investigate the changes in arc and ring values for the defined target, the trajectory of the electrode was also created in the software (Figure 1C). We skipped the normal step of identifying and avoiding cerebral vasculature using contrast enhanced T1-weigthed MRI because it was not applicable in this simulation setting.

Firstly, the MRI T2 was chosen as registration series and co-registered with all seven CT series (Figure 2C). The fiducials of the N-localizer on the stereotactic CT images were registered to the planning software automatically for each merged series (Figure 2D). The stereotactic coordinates including X, Y, Z, Ring and Arc angles generated for the target point for seven merged series were recorded. Then, the opposite was applied in order to test all different combinations. Seven CT series were chosen as registration series one by one and merged with T2 series after registration (images not shown). The stereotactic coordinates generated for the target point for seven merged series were recorded as well.

The image co-registrations were processed with the auto-merge algorithm of the software and the results were checked for each merged series visually as is standard clinical practice. Auto-merge algorithm of planning station was successful for all merged image groups (Figure 2C).

\section{Results}

The stereotactic coordinates generated by the planning station for the previously defined target and trajectory are shared in Table 1. The total number of images, stereotactic coordinates and trajectory angle values related to the target for each CT series are also shared in Table 1.

The number of CT images obtained for seven stereotactic CT scans with $0.5 \mathrm{~mm}, 1 \mathrm{~mm}, 1.5 \mathrm{~mm}, 2 \mathrm{~mm}, 3 \mathrm{~mm}$, $4 \mathrm{~mm}$ and 5mm slice thicknesses were 614, 263, 184, 123, 92, 62 and 46 respectively. The number of the slices obtained with $2 \mathrm{~mm}$ slice thickness for the non-stereotactic T2 weighted MRI was 72.

For the co-registration in which MR T2 series were assigned as registration series, the average $\mathrm{X}, \mathrm{Y}, \mathrm{Z}$ coordinates, ring and arc angles were 102.96 (standard deviation $[\mathrm{SD}] \pm 0.1 \mathrm{~mm}), 106.89(\mathrm{SD} \pm 0.03 \mathrm{~mm})$, $146.80(\mathrm{SD} \pm 0.05 \mathrm{~mm}), 76.20\left(\mathrm{SD} \pm 0.0^{\circ}\right), 104.81\left(\mathrm{SD} \pm 0.3^{\circ}\right)$ respectively. The calculated absolute differences between maximum and minimum recorded values for $\mathrm{X}, \mathrm{Y}, \mathrm{Z}$ coordinates, ring and arc angles were $0.3 \mathrm{~mm}$, $0.1 \mathrm{~mm}, 0.2 \mathrm{~mm}, 0^{\circ}$ and $0.1^{\circ}$ respectively.

For the co-registration process in which the stereotactic CT's were assigned as registration series, the average X, $\mathrm{Y}, \mathrm{Z}$ coordinates, ring and arc angles were $102.93(\mathrm{SD} \pm 0.1 \mathrm{~mm}), 106.83(\mathrm{SD} \pm 0.07 \mathrm{~mm}), 146.97(\mathrm{SD} \pm 0.12 \mathrm{~mm})$, $76.04\left(\mathrm{SD} \pm 0.1^{\circ}\right), 104.73\left(\mathrm{SD} \pm 0.07^{\circ}\right)$ respectively. The calculated absolute differences between maximum and minimum recorded values for $\mathrm{X}, \mathrm{Y}, \mathrm{Z}$ coordinates, ring and arc angles were $0.3 \mathrm{~mm}, 0.2 \mathrm{~mm}, 0.4 \mathrm{~mm}, 0.3^{\circ}$ and $0.2^{\circ}$ respectively. 


\begin{tabular}{|c|c|c|c|c|c|c|c|c|c|c|c|c|}
\hline \multirow{3}{*}{ Imaging Modality } & \multirow{3}{*}{$\begin{array}{l}\text { Slice Thicknesses of } \\
\text { the Scans }(\mathrm{mm})\end{array}$} & \multirow{3}{*}{$\begin{array}{c}\text { Number of Images in } \\
\text { the Scans }\end{array}$} & \multicolumn{5}{|c|}{ Registration Series: T2 } & \multicolumn{5}{|c|}{ Registration Series: CT } \\
\hline & & & \multicolumn{3}{|c|}{ Coordinates (mm) } & \multicolumn{2}{|c|}{ Angles $\left({ }^{\circ}\right)$} & \multicolumn{3}{|c|}{ Coordinates (mm) } & \multicolumn{2}{|c|}{ Angles $\left({ }^{\circ}\right)$} \\
\hline & & & $\mathrm{x}$ & $\mathbf{Y}$ & $Z$ & Ring & Arc & $\mathrm{x}$ & $\mathbf{Y}$ & Z & Ring & Arc \\
\hline \multirow{7}{*}{ Stereotactic CT } & 0.50 & 614 & 102.7 & 106.9 & 146.7 & 76.2 & 104.8 & 102.7 & 106.9 & 146.7 & 76.2 & 104.8 \\
\hline & 1.00 & 263 & 103 & 106.9 & 146.8 & 76.2 & 104.8 & 103 & 106.9 & 147 & 76.2 & 104.7 \\
\hline & 1.50 & 184 & 103 & 106.9 & 146.8 & 76.2 & 104.8 & 103 & 106.8 & 147 & 75.9 & 104.7 \\
\hline & 2.00 & 123 & 103 & 106.9 & 146.8 & 76.2 & 104.8 & 103 & 106.8 & 147 & 76 & 104.6 \\
\hline & 3.00 & 92 & 103 & 106.9 & 146.8 & 76.2 & 104.8 & 103 & 106.8 & 147 & 76 & 104.7 \\
\hline & 4.00 & 62 & 103 & 106.9 & 146.8 & 76.2 & 104.8 & 102.9 & 106.9 & 147 & 76 & 104.8 \\
\hline & 5.00 & 46 & 103 & 106.8 & 146.9 & 76.2 & 104.9 & 102.9 & 106.7 & 147.1 & 76 & 104.8 \\
\hline Non Stereotactic MR & 2.00 & 72 & \multicolumn{5}{|c|}{ Registration Series: T2 } & \multicolumn{5}{|c|}{ Registration Series: CT } \\
\hline \multicolumn{3}{|c|}{ Average } & 102.96 & 106.89 & 146.80 & 76.20 & 104.81 & 102.93 & 106.83 & 146.97 & 76.04 & 104.73 \\
\hline \multicolumn{3}{|c|}{ Standard Dev. } & 0.10 & 0.03 & 0.05 & 0.00 & 0.03 & 0.10 & 0.07 & 0.12 & 0.10 & 0.07 \\
\hline \multicolumn{3}{|c|}{ Absolute Difference $(\mathrm{mm})$} & 0.3 & 0.1 & 0.2 & 0 & 0.1 & 0.3 & 0.2 & 0.4 & 0.3 & 0.2 \\
\hline
\end{tabular}

Table 1: X, Y, Z coordinates; ring and arc angles of the defined target for each CT scan are presented in table 1. Number of slices, slice thicknesses values for each CT and MR series are presented in table 1 too. The results were shared under the name of two different registration series (T2 and CT) where these images were chosen as registration series for target calculations. The average coordinate, standard deviation and absolute differences between the highest and the lowest coordinate/angle $(\Delta)$ values for each CT series are also shared in Table 1.

\section{Discussion}

The results of our study show that increasing the slice thickness of the stereotactic CT, up to 5mm, has no significant effect on the stereotactic calculations with absolute differences in all scenario's less than $0.5 \mathrm{~mm}$. A $£ 1 \mathrm{~mm}$ deviation is well accepted for clinical use in stereotactic neurosurgical procedures ${ }^{6,13}$.

Comparing our results with reported stereotactic errors in the literature confirms our finding. Reported mean absolute errors for the mediolateral axis/X coordinate are between $0.11 \mathrm{~mm}(\mathrm{SD} \pm 0.78)$ and $0.61 \mathrm{~mm}(\mathrm{SD} \pm$ $022)$ in a review comparing different kinds of stereotactic software packages ${ }^{13}$. Two different studies report $1 \mathrm{~mm}(\mathrm{SD} \pm 0.7)$ and $1.4 \mathrm{~mm}(\mathrm{SD} \pm 1.0)$ absolute error respectively ${ }^{13,18,19}$. Our mean absolute error for the $\mathrm{X}$ coordinate of $0.3 \mathrm{~mm}(\mathrm{SD} \pm 0.1)$ is comparable to the highest reported error. The same studies reported mean absolute errors for anteroposterior axis/Y coordinates between $0.33(\mathrm{SD} \pm 0.26)$ and $1.10(\mathrm{SD} \pm 020), 0.9 \mathrm{~mm}$ $(\mathrm{SD} \pm 0.5)$ and $1.2 \mathrm{~mm}(\mathrm{SD} \pm 1.0)[18,19,13]$. Our result of $0.15 \mathrm{~mm}(\mathrm{SD} \pm 0.07)$ showed an even higher accuracy. Regarding the superoinferior axis/Z-coordinate reported accuracies in the literature vary and this stereotactic coordinate is notorious for having the highest errors. We found a mean absolute error of $0.3 \mathrm{~mm}$ $(\mathrm{SD} \pm 0.12$ ) comparable to or even lower than results in the literature of $0.295 \mathrm{~mm}$ and between $0.46 \mathrm{~mm}$ (SD $\pm 0.55)$ and $1.4 \mathrm{~mm}(\mathrm{SD} \pm 0.98)$ respectively ${ }^{5,13}$. An older study reported a mean absolute error up to $8 \mathrm{~mm}$ and the two former mentioned studies found mean absolute errors of $0.7 \mathrm{~mm}(\mathrm{SD} \pm 0.6), 1.3 \mathrm{~mm}(\mathrm{SD} \pm 0.9)$ respectively $\mathrm{y}^{7,18,19}[7,18,19]$. Although, values for the $\mathrm{Z}$ coordinate differ more than for the $\mathrm{X}$ and $\mathrm{Z}$ coordinate, our results are within the highest reported stereotactic errors in the literature.

In our former study regarding frame mounting and stereotactic accuracy we reported a relative high absolute difference for the $\mathrm{Z}$ coordinate of $2.46 \mathrm{~mm}^{6}$. However, for the software calculations alone this deviation decreased to $0.9 \mathrm{~mm}$. We concluded that manual calculations can lead to stereotactic inaccuracy regarding the $\mathrm{Z}$ coordinate. As stated in the introduction this strengthened our opinion that, with the widely available and userfriendly software packages, manual calculations no longer have an important role in stereotaxy. Remarkably, the former mentioned review of Geevarghese et al found a significant difference in the Z-axis error $(0.46 \mathrm{~mm} \mathrm{SD} \pm$ $0.55)$ compared to the $\mathrm{X}$ - axis $(0.25 \mathrm{~mm} \mathrm{SD} \pm 0.15)$ and $\mathrm{Y}$-axis $(0.33 \mathrm{~mm} \mathrm{SD} \pm 0.26)^{13}$. However, this finding is in accordance with the literature but their hypothesized explanation was the slice thickness of MRI $(1,5 \mathrm{~mm})$ and CT $(1,25 \mathrm{~mm})$. A proposed decrease in slice thickness of both image modalities could improve accuracy in MRI/CT fusion. In contrast, our results show no significant change in stereotactic (fusion) calculations with increasing slice thickness in CT. The question about MRI slice thickness in stereotaxy will be addressed below. 
Additionally, we investigated the changes in ring and arc angles with the increase in slice thickness too. The mean absolute errors calculated for ring and arc angles of the Leksell stereotactic system were $0.15 \mathrm{~mm}$ (SD $\pm 0.1)$ and $0.15 \mathrm{~mm}(\mathrm{SD} \pm 0.07)$ respectively. As far as we know, there were no available studies or published data questioning the stereotactic calculations through trajectory angles. Despite no other results to compare our findings, we are of the opinion that these very small deviations do not seem to affect the stereotactic calculation of ring and arc angle components of the trajectory.

Even though, our mean absolute errors for all stereotactic coordinates are within the lowest range reported in the literature we should consider they might not arise from the CT slice thickness alone. Stereotaxy has more potential source for error including the mechanical accuracy of the stereotactic system itself and all the steps of the surgical procedure (image techniques e.g. CT slice thickness, registration, mechanical connections, potential fiducial marker defects and system adjustments) together called the application accuracy ${ }^{20,21}$. However, this is out of the scope of this paper but it is good to keep in mind. There are very few studies in the literature focusing on the effect of CT systems on stereotactic accuracy. Tafreshi et al., examined the stereotactic accuracy between 2 CT scanners with both helical and single-slice image acquisition using a phantom. They concluded that single-slice image acquisition CT systems were superior in terms of stereotactic accuracy and interscanner accuracy was deviant, interesting also in the Z-axis ${ }^{22}$. Our study used a helical imaging protocol which we could have compared with a single slice image acquisition protocol. However, our results still show that it is safe and feasible to acquire stereotactic coordinates from stereotactic CT images with higher slice thickness values if image fusion is performed with a thin-sliced MR series, even with a helical image acquisition. Nevertheless, it is valuable for the literature to consider and investigate CT parameters which could affect stereotactic accuracy.

The radiological information concerning MRI required to perform DBS surgeries has been investigated thoroughly. Several publications detailing how to achieve the highest image quality and surgical accuracy recommend thin sliced MR images $(£ 2 \mathrm{~mm})^{23}, 24$. The optimum localization of the desired targets passes through the use of thin-slice contiguous MR images ${ }^{4}$.

Additionally, we published that the use of T2W MR images with $2 \mathrm{~mm}$ slice thickness is radiologically correlated with $1 \mathrm{~mm}$ step-sized Microelectrode Recordings (MER) of the Subthalamic Nucleus (STN) ${ }^{25}$. This correlation supports the need for the thin slice MR images in DBS surgeries where high accuracy is required to achieve accurate localization. Moreover, the use of MR images with thicker slices will worsen the image reconstruction quality in different imaging planes. With this worsening effect, targeting the anatomical structures like STN and Globus Pallidus interna (GPi) will not be accurate since the boundaries of these anatomical structures will not be visualized precisely through these reconstructed images ${ }^{26}$. Lastly, the localization and registration of important landmarks like Anterior commissure (AC) and Posterior Commissure (PC) which is a crucial step for the use of stereotactic planning software, could be performed more precisely with the use of thin slice MR images. Thus, thin sliced contiguous MRI is mandatory in stereotaxy. The arising question if thinner sliced MRI (even less than $1.5 \mathrm{~mm}$ ) could improve the stereotactic accuracy as mentioned above is an interesting thought but beyond the aim of this article.

In the early stages of stereotaxy, CT was often the only available imaging modality for surgeries related to movement disorders ${ }^{27}$. Today, MRI has replaced CT as imaging modality of choice for planning and targeting for obvious reasons. CT is only required to create the "stereotactic MRI". (The postoperative complication check is not considered as a part of the stereotactic procedure at this point). CT technologies have advanced enormously, including scanning time, making it more and more convenient to use ${ }^{28}$. However, CT delivers intensive ionizing radiation when compared to MRI. Moreover, the increase in image acquisition speed also increases ionizing radiation exposure. Because of this, the more sophisticated use of CT could lead to an increase in exposure of ionizing radiation ${ }^{29}$. 
In the routine clinical application, MRI and $\mathrm{CT}$ images are requested with the highest possible quality and resolution with the idea to perform the best practice. However, overexposed or oversized scans do not necessarily contain more diagnostic or useful information then required ${ }^{30}$. It is a well-known fact that with the use of smaller slice thickness values within CT scanners, the exposed ionized radiation dose will also consequently increase ${ }^{31}$. With the wide availability and use of CT the aim should be find the balance in getting the best clinical useable images with the least amount of ionizing radiation.

Nowadays, new imaging modalities like O-arm and intraoperative CT machines have been introduced to the market in order to provide radiological information inside the operating room $^{32,33}$. These technologies are innovative since the information required for stereotactic image registration is no more dependent of traditional fixed CT machines. Also, the image resolution and image quality of these are comparable and as accurate as the classic imaging modalities ${ }^{34}$. Furthermore, this information is available with diminished ionized radiation exposure in comparison to traditional CT machines ${ }^{34,35}$. This non-inferiority together with mobility and less radiation makes these novel imaging techniques very interesting in the stereotaxy.

Lastly, the use of unnecessary small slices in radiological scanning protocols could increase the number of images generated by the image processing tools (Table 1). This might lead to image fusion failures or slow image processing during the planning phase of stereotaxy ${ }^{36}$.

In this study we analyzed seven CT scans with different slice thicknesses and our results showed that increasing slice thickness has no significant effect on stereotactic measurements when merged with a thin-sliced (2mm) contiguous MRI series. The fused images of all combinations, including using the stereotactic CT or MRI as registration series, resulted in very similar and accurate stereotactic coordinates, arc and ring settings. If thick-sliced CT is non-inferior to thin-sliced CT in providing the stereotactical information needed for coregistration with MRI in procedures like DBS, this could be an interesting alternative to explore.

\section{Conclusion}

We found no significant effect of increasing slice thickness of CT on the stereotactic calculations and its accuracy when co-registered with a thin-sliced MRI in a simulated stereotactic DBS procedure. More slices do not always add useful extra diagnostic information needed for these procedures. More research should be done to look into the clinical applicability of thick sliced CT in a stereotactical setting and its effect on ionized radiation exposure. 


\section{References}

1. Guo Z, Leong MC-W, Su H, Kwok K-W, Chan DT-M, Poon W-S. Techniques for stereotactic neurosurgery: beyond the frame, toward the intraoperative magnetic resonance imaging-guided and robot-assisted approaches. World Neurosurgery. 2018;116:77-87.

2. Lemieux L, Jagoe R. Effect of fiducial marker localization on stereotactic target coordinate calculation in CT slices and radiographs. Physics in Medicine \& Biology. 1994;39(11):1915.

3. Sharan AD, Andrews DW. Stereotactic frames: technical considerations. NEUROLOGICAL DISEASE AND THERAPY. 2003;58:11-20.

4. Zrinzo L. Pitfalls in precision stereotactic surgery. Surgical neurology international. 2012;3(Suppl 1):S53.

5. Montoya AT, Pereira J. Testing the precision of stereotactic planning systems. Computerized medical imaging and graphics. 1998;22(4):317-21.

6. Alptekin O, Gubler FS, Ackermans L, et al. Stereotactic accuracy and frame mounting: A phantom study. Surgical Neurology International. 2019;10.

7. Walton L, Hampshire A, Forster DM, Kemeny AA. A phantom study to assess the accuracy of stereotactic localization, using T1-weighted magnetic resonance imaging with the Leksell stereotactic system. Neurosurgery. 1996;38(1):170-8.

8. Rezai AR, Kopell BH, Gross RE, et al. Deep brain stimulation for Parkinson's disease: surgical issues. Movement disorders: official journal of the Movement Disorder Society. 2006;21(S14):S197-S218.

9. Jonker BP. Image fusion pitfalls for cranial radiosurgery. Surgical Neurology International. 2013;4(Suppl 3):S123.

10. Bronen RA, Fulbright RK, Spencer DD, et al. Refractory epilepsy: comparison of MR imaging, CT, and histopathologic findings in 117 patients. Radiology. 1996;201(1):97-105.

11. Bronen RA, Fulbright RK, Spencer SS, Spencer DD, Kim JH, Lange RC. Economic impact of replacing CT with MR imaging for refractory epilepsy. Magnetic resonance imaging. 1997;15(7):857-62.

12. Stoffner R, Augschöll C, Widmann G, Böhler D, Bale R, editors. Accuracy and feasibility of frameless stereotactic and robot-assisted CT-based puncture in interventional radiology: a comparative phantom study. RöFo-Fortschritte auf dem Gebiet der Röntgenstrahlen und der bildgebenden Verfahren; 2009: (C) Georg Thieme Verlag KG Stuttgart· New York.

13. Geevarghese R, Tuura ROG, Lumsden DE, Samuel M, Ashkan K. Registration accuracy of CT/MRI fusion for localisation of deep brain stimulation electrode position: an imaging study and systematic review. Stereotactic and functional neurosurgery. 2016;94(3):159-63.

14. Bradac O, Steklacova A, Nebrenska K, Vrana J, de Lacy P, Benes V. Accuracy of VarioGuide frameless stereotactic system against frame-based stereotaxy: prospective, randomized, single-center study. World Neurosurgery. 2017;104:831-40.

15. Lunsford L, Latchaw RE, Vries J. Stereotactic implantation of deep brain electrodes using computed tomography. Neurosurgery. 1983;13(3):280-6.

16. Rizzone M, Lanotte M, Bergamasco B, et al. Deep brain stimulation of the subthalamic nucleus in Parkinson's disease: effects of variation in stimulation parameters. Journal of Neurology, Neurosurgery \& Psychiatry. 2001;71(2):215-9.

17. Ford JM, Decker SJ. Computed tomography slice thickness and its effects on three-dimensional reconstruction of anatomical structures. Journal of Forensic Radiology and Imaging. 2016;4:43-6.

18. Sharma M, Rhiew R, Deogaonkar M, Rezai A, Boulis N. Accuracy and precision of targeting using frameless stereotactic system in deep brain stimulator implantation surgery. Neurology India. 2014;62(5):503.

19. Bot M, Van Den Munckhof P, Bakay R, Sierens D, Stebbins G, Metman LV. Analysis of stereotactic accuracy in patients undergoing deep brain stimulation using Nexframe and the Leksell frame. Stereotactic and functional neurosurgery. 2015;93(5):316-25.

20. Maciunas RJ, Galloway Jr RL, Latimer JW. The application accuracy of stereotactic frames. Neurosurgery. 1994;35(4):682-95.

21. Alptekin O, Kocabicak E, Gubler FS, Ackermans L, Kubben PL, Temel Y. Perioperative technical complications in deep brain stimulation surgeries. Turkish neurosurgery. 2018;28(3):483-9.

22. Tafreshi AR, Peng T, Yu C, et al. A phantom study of the spatial precision and accuracy of stereotactic localization using computed tomographic imaging with the Leksell stereotactic system. World Neurosurgery. 2020.

23. Hirabayashi H, Tengvar M, Hariz MI. Stereotactic imaging of the pallidal target. Movement disorders: official journal of the Movement Disorder Society. 2002;17(S3):S130-S4.

24. Dormont D, Ricciardi KG, Tandé D, et al. Is the subthalamic nucleus hypointense on T2-weighted images? A correlation study using MR imaging and stereotactic atlas data. American Journal of Neuroradiology. 2004;25(9):1516-23.

25. Kocabicak E, Aygun D, Ozaydın I, et al. Does probe's eye subthalamic nucleus length on T2W MRI correspond with microelectrode recording in patients with deep brain stimulation for advanced Parkinson's disease? Turkish neurosurgery. 2013;23(5):658-65.

26. Sudhyadhom A, Haq IU, Foote KD, Okun MS, Bova FJ. A high resolution and high contrast MRI for differentiation of subcortical structures for DBS targeting: the Fast Gray Matter Acquisition T1 Inversion Recovery (FGATIR).

Neuroimage. 2009;47:T44-T52.

27. Gildenberg PL. Whatever happened to stereotactic surgery? Neurosurgery. 1987;20(6):983-7.

28. Kalender WA. X-ray computed tomography. Physics in Medicine \& Biology. 2006;51(13):R29.

29. Smith-Bindman R, Lipson J, Marcus R, et al. Radiation dose associated with common computed tomography examinations and the associated lifetime attributable risk of cancer. Archives of internal medicine. 2009;169(22):2078-86.

30. Tsalafoutas IA, Koukourakis GV. Patient dose considerations in computed tomography examinations. World journal of radiology. 2010;2(7):262. 
31. Raman SP, Mahesh M, Blasko RV, Fishman EK. CT scan parameters and radiation dose: practical advice for radiologists. Journal of the American College of Radiology. 2013;10(11):840-6.

32. Katisko JPA, Kauppinen MT, Koivukangas JP, Heikkinen ER. Stereotactic operations using the O-arm. Stereotactic and functional neurosurgery. 2012;90(6):401-9.

33. Sharma M, Deogaonkar M. Accuracy and safety of targeting using intraoperative "O-arm" during placement of deep brain stimulation electrodes without electrophysiological recordings. Journal of Clinical Neuroscience. 2016;27:80-6.

34. Carlson JD. Stereotactic registration using cone-beam computed tomography. Clinical neurology and neurosurgery. 2019.

35. Pitteloud N, Gamulin A, Barea C, Damet J, Racloz G, Sans-Merce M. Radiation exposure using the O-arm ${ }^{\circledR}$ surgical imaging system. European spine journal. 2017;26(3):651-7.

36. Rocha LGSd, Amaro Junior E. Seeking tools for image fusion between computed tomography, structural and functional magnetic resonance methods for applications in neurosurgery. Einstein (São Paulo). 2012;10(2):151-7. 


\section{Chapter 5}

Is there still need for microelectrode recording now the subthalamic nucleus can be

\section{well visualized with high field and ultrahigh MR imaging?}

Kocabicak, E., Alptekin, O., Ackermans, L., Kubben, P., Kuijf, M., Kurt, E., Esselink, R., Temel, Y. (2015).

Is there still need for microelectrode recording now the subthalamic nucleus can be well visualized with high field and ultrahigh MR imaging? Frontiers in integrative neuroscience, 9, 46. 


\section{The Question}

High frequency stimulation of the subthalamic nucleus (STN) is an effective treatment for patients with Parkinson's disease (PD) ${ }^{1-3}$. The technique has been further refined throughout the years by improved magnetic resonance imaging (MRI) techniques, advanced neurophysiological recording possibilities, and advances in hardware and software technology ${ }^{2}$. There are at least two major determining factors for an acceptable therapeutic outcome: patient selection ${ }^{4}$ and the accuracy of targeting of the relatively small STN ${ }^{5}$. The latter requires a state-of-the art stereotactic approach, adequate imaging facilities, and a detailed neurophysiological mapping of the target area. The preferred area within the STN is the motor part (thought to be located dorsolaterally in the STN), which can, be to some extent, identified by intra operative multi-unit activity analyses, and MRI based tractography ${ }^{6,7}$. While the STN could not be visualized on MRI images when modern DBS of the STN surgeries started in Grenoble in 1993, nowadays its visualization has become a routine procedure for most centers offering DBS for patients with PD. While using intraoperative electrophysiology was evident in the beginning, now it is questioned whether it still has an essential added value. In this opinion article, we aim to provide an answer on the question whether or not electrophysiology still has a clinically relevant role in this era of advanced neuroimaging technology, which enables us to visualize both function and structure anatomy.

\section{Old Debate}

The discussion of whether or not to use intraoperative microelectrode recording (MER) is not a new one ${ }^{8}$. This discussion was perhaps less vivid when modern DBS started to be applied in patients with PD. The STN was an invisible target on MR images in most centers and MER was considered very helpful to find and delineate the boundaries of the target ${ }^{9-11}$. Since then things have changed. However, currently the STN can be directly visualized on T2 weighed and susceptibility weighed MR images.

The imaging field progresses rapidly further with ultra-high field imaging modalities becoming now available for patients ${ }^{12}$. It is more than 15 years ago that that the visualization of the STN for DBS surgeries was described ${ }^{13}$. Mostly, T2 weighed and inversion recovery MRI sequences have been used. In most of the patients, the predefined target on T2 weighed MR images was chosen for implantation after intra-operative electrophysiology and test stimulation ${ }^{14-16}$. This meant that in most patients MRI images could reliably show the STN, except for the y axis, in which microelectrode recording (MER) indicated that the STN extended more anteriorly than suggested by MRI ${ }^{17}$. Detailed volumetric analysis of MER determined borders of the STN and MRI- defined borders in 22 patients (44 STN's), showed that MER-determined borders of the STN were exceeding the MRI signal ${ }^{18}$. In addition, we examined the entry and exit borders of the STN on MRI images and with MER, using the probe's eye trajectory ${ }^{19}$.We found that T2 weighed MRI could reliably predict the electrophysiological entry and exit of the STN. Although these data confirm the accuracy of MRI in visualizing the STN, there are also limitations. There are known variations between the patients with respect to the $\mathrm{x}, \mathrm{y}$, and $\mathrm{z}$ planes, and the borders can sometimes be less clear, mainly toward the substantia nigra pars reticulata $(\mathrm{SNr})^{17,19}$. 


\section{From Atlas Based to MRI Based Coordinates and from Single Electrode to Multiple Electrode Recordings}

In our previous series of 55 patients with PD who underwent DBS of the STN, atlas-based coordinates were used and in about one third of the patients the predefined target (central trajectory) was used for final electrode implantation, after MER and intra-operative test stimulation ${ }^{20}$. With applying individually adjusted coordinates based on T2 weighed MRI, the central trajectory was chosen in about two-thirds of the patients ${ }^{19}$, ${ }^{21}$. This has resulted in a clear reduction in operation time. Similar rates have been reported by others with atlas based ${ }^{22}$ and MRI-based targeting coordinates ${ }^{23}$. The change from 1.5 to $3.0 \mathrm{~T}$ has also improved the accuracy of targeting ${ }^{24,25}$. Another development has been the change of single-electrode to multiple-electrode intra-operative electrophysiological recordings ${ }^{20}$. The latter provides more detailed information about the electrophysiological boundaries of the STN; however, implantation of several electrodes at one time might increase the risk of bleeding. We found that the simultaneous implantation of multiple electrodes did not cause more bleedings or other major intracranial complication. The use of multiple electrodes resulted in better motor results when compared with patients who underwent DBS of the STN guided with a single recording electrode. There are reports, however, suggesting increased risk of hemorrhage due to MER ${ }^{26,27}$.

\section{Back to the Question}

Is intra-operative electrophysiology necessary to find the STN? Our answer is no based on the advances in MRI technology. In line with this experienced DBS centers have shown good outcome with a MRI-guided approach $^{28,29}$. So, should we abandon MER then? In our centers, we have decided not to abandon it for a number of reasons. Even in experienced centers, in about two-thirds of the cases, the predefined target is chosen for final implantation. In one-third, an alternative trajectory is needed. With MER, alternative trajectories are immediately available. The trajectory with the second longest and, if needed, the third longest STN activity can be used as alternative trajectories. Two other less common reasons to use intra-operative electrophysiology can be an unexpected error in the stereotactic approach or a shift caused by excessive CSF leakage or a hematoma ${ }^{23}$. 


\section{References}

1. Odekerken VJ, van Laar T, Staal MJ, et al. Subthalamic nucleus versus globus pallidus bilateral deep brain stimulation for advanced Parkinson's disease (NSTAPS study): a randomised controlled trial. The Lancet Neurology. 2013;12(1):37-44.

2. Kocabicak E, Temel Y. Deep brain stimulation of the subthalamic nucleus in Parkinson's disease: surgical technique, tips, tricks and complications. Clinical neurology and neurosurgery. 2013;115(11):2318-23.

3. Schuepbach W, Rau J, Knudsen K, et al. Neurostimulation for Parkinson's disease with early motor complications. New England Journal of Medicine. 2013;368(7):610-22.

4. Deuschl G, Schade-Brittinger C, Krack P, et al. Neurostimulation Section. A randomized trial of deep-brain stimulation for Parkinson's disease N Engl J Med. 2006;355:896-908.

5. Temel Y, Blokland A, Steinbusch HW, Visser-Vandewalle V. The functional role of the subthalamic nucleus in cognitive and limbic circuits. Progress in neurobiology. 2005;76(6):393-413.

6. Zaidel A, Spivak A, Grieb B, Bergman H, Israel Z. Subthalamic span of $\beta$ oscillations predicts deep brain stimulation efficacy for patients with Parkinson's disease. Brain. 2010;133(7):2007-21.

7. Brunenberg EJ, Platel B, Hofman PA, ter Haar Romeny BM, Visser-Vandewalle V. Magnetic resonance imaging techniques for visualization of the subthalamic nucleus: a review. Journal of neurosurgery. 2011;115(5):971-84.

8. Hariz MI. Safety and risk of microelectrode recording in surgery for movement disorders. Stereotactic and functional neurosurgery. 2002;78(3-4):146-57.

9. Pollak P, Benabid A, Gross C, et al. Effects of the stimulation of the subthalamic nucleus in Parkinson disease. Revue neurologique. 1993;149(3):175-6.

10. Limousin P, Pollak P. Benazzouz a, Hoffmann D, Le Bas JF, Broussolle E, et al. Effect of parkinsonian signs and symptoms of bilateral subthalamic nucleus stimulation Lancet. 1995;345:0140-6736.

11. Shamir RR, Zaidel A, Joskowicz L, Bergman H, Israel Z. Microelectrode recording duration and spatial density constraints for automatic targeting of the subthalamic nucleus. Stereotactic and functional neurosurgery. 2012;90(5):325-34.

12. Plantinga BR, Temel Y, Roebroeck A, et al. Ultra-high field magnetic resonance imaging of the basal ganglia and related structures. Frontiers in human neuroscience. 2014;8:876.

13. Starr PA, Vitek JL, DeLong M, Bakay RA. Magnetic resonance imaging-based stereotactic localization of the globus pallidus and subthalamic nucleus. Neurosurgery. 1999;44(2):303-13.

14. Bejjani B-P, Dormont D, Pidoux B, et al. Bilateral subthalamic stimulation for Parkinson's disease by using three-dimensional stereotactic magnetic resonance imaging and electrophysiological guidance. Journal of neurosurgery. 2000;92(4):615-25.

15. Egidi M, Rampini P, Locatelli M, et al. Visualisation of the subthalamic nucleus: a multiple sequential image fusion (MuSIF) technique for direct stereotaxic localisation and postoperative control. Neurological Sciences. 2002;23(2):s71-s2.

16. Starr P. Chris tine CW, Theodosopoulos PV, Lindsey N, Byrd D, Mosley A, Marks WJ Jr. Im plan ta tion of deep brain stimu la tors into the subthalamic nu cleus: tech nical ap proach and mag netic res o nance im ag ing-ver ified lead lo ca tions J Neurosurg. 2002;97:370-87.

17. Hamani C, Richter EO, Andrade-Souza Y, Hutchison W, Saint-Cyr JA, Lozano AM. Correspondence of microelectrode mapping with magnetic resonance imaging for subthalamic nucleus procedures. Surgical neurology. 2005;63(3):249-53.

18. Schlaier JR, Habermeyer C, Warnat J, et al. Discrepancies between the MRI-and the electrophysiologically defined subthalamic nucleus. Acta neurochirurgica. 2011;153(12):2307-18.

19. Kocabicak E, Aygun D, Ozaydin I, et al. Does probe's eye subthalamic nucleus length on T2W MRI correspond with microelectrode recording in patients with deep brain stimulation for advanced Parkinson's disease? Turkish neurosurgery. 2013;23(5):658-65.

20. Temel Y, Wilbrink P, Duits A, et al. Single electrode and multiple electrode guided electrical stimulation of the subthalamic nucleus in advanced Parkinson's disease. Operative Neurosurgery. 2007;61(suppl_5):ONS346-ONS57.

21. Tonge M, Kocabıçak E, Ackermans L, Kuijf M, Temel Y. Final electrode position in subthalamic nucleus deep brain stimulation surgery: a comparison of indirect and direct targeting methods. 2016.

22. Amirnovin R, Williams ZM, Cosgrove GR, Eskandar EN. Experience with microelectrode guided subthalamic nucleus deep brain stimulation. Operative Neurosurgery. 2006;58(suppl_1):ONS-96-ONS-102.

23. Reck C, Maarouf M, Wojtecki L, et al. Clinical outcome of subthalamic stimulation in Parkinson's disease is improved by intraoperative multiple trajectories microelectrode recording. Journal of Neurological Surgery Part A: Central European Neurosurgery. 2012;73(06):377-86.

24. Toda H, Sawamoto N, Hanakawa T, et al. A novel composite targeting method using high-field magnetic resonance imaging for subthalamic nucleus deep brain stimulation. Journal of neurosurgery. 2009;111(4):737-45.

25. Kerl HU, Gerigk L, Pechlivanis I, Al-Zghloul M, Groden C, Nölte I. The subthalamic nucleus at 3.0 Tesla: choice of optimal sequence and orientation for deep brain stimulation using a standard installation protocol. Journal of neurosurgery. 2012;117(6):1155-65.

26. Ben-Haim S, Asaad WF, Gale JT, Eskandar EN. Risk factors for hemorrhage during microelectrode-guided deep brain stimulation and the introduction of an improved microelectrode design. Neurosurgery. 2009;64(4):754-63.

27. Xiaowu H, Xiufeng J, Xiaoping Z, et al. Risks of intracranial hemorrhage in patients with Parkinson's disease receiving deep brain stimulation and ablation. Parkinsonism \& related disorders. 2010;16(2):96-100.

28. Ostrem JL, Galifianakis NB, Markun LC, et al. Clinical outcomes of PD patients having bilateral STN DBS using high-field interventional MR-imaging for lead placement. Clinical neurology and neurosurgery. 2013;115(6):708-12.

29. Aviles-Olmos I, Kefalopoulou Z, Tripoliti E, et al. Long-term outcome of subthalamic nucleus deep brain stimulation for Parkinson's disease using an MRI-guided and MRI-verified approach. Journal of Neurology, Neurosurgery \& Psychiatry. 2014;85(12):1419-25. 


\title{
Chapter 6
}

Microelectrode recording for deep brain stimulation of the subthalamic nucleus in patients with advanced Parkinson's disease: Advantage or loss of time?

\author{
Alptekin, O., Kocabicak, E., Aygun, D., Yildiz, O., Temel, Y. (2019).
}

Microelectrode Recording for Deep Brain Stimulation of the Subthalamic Nucleus in Patients with Advanced Parkinson's Disease: Advantage or Loss of Time? Turkish Neurosurgery, 29(5), 677-682. 


\begin{abstract}
Background: Deep brain stimulation (DBS) of the subthalamic nucleus in patients with Parkinson's disease (PD) can be performed with or without microelectrode recording (MER). However, MER increases the duration of the operation. Here, we have investigated the effect of using MER on the length of time required to carry out a DBS procedure.
\end{abstract}

Materials and methods: The time required to include MER in the DBS operation was calculated for the first and second sides in 24 patients with PD. The number of microelectrodes used on each trajectory for the first and second sides, and the percentage of permanent electrodes implanted on each trajectory for the first and second sides, were quantified.

Results: The average times taken to use MER were $23.4 \pm 6.2 \mathrm{~min} ., 17.4 \pm 6.5 \mathrm{~min}$., and $41.2 \pm 6.3 \mathrm{~min}$ for the first side, second side and total procedure, respectively. In $75 \%$ of patients, the permanent electrode was implanted at the planned target site for the first side and in $61 \%$ of patients for the second side.

Conclusions: MER extends the time required to carry out the DBS procedure. However, during surgery, it provides real-time information on the electrodes' neurophysiological locations and helps the surgical team choose an alternative target if the planned target does not produce satisfying results. 


\section{Introduction}

Deep brain stimulation (DBS) of the subthalamic nucleus (STN) has become a well-accepted treatment for patients with advanced Parkinson's disease (PD) ${ }^{1-3}$. It is well-known that the success of DBS depends on the accuracy in targeting the $\mathrm{STN}^{4}$. Imaging techniques, stereotactic precision, and intraoperative microelectrode recording (MER) procedures help with precise targeting. Historically, the STN was not detectable using magnetic resonance imaging (MRI) for DBS; therefore, using intraoperative MER was essential ${ }^{5}$. However, with advancements in MRI, particularly the development of T2-weighed images, the STN can be observed directly. Using this improved technology, centers began to apply direct targeting methods and rejected MER. There are two main reasons why centers stopped using MER. First, there is an apparent risk of increased bleeding, although some reports claim the opposite ${ }^{6-12}$. Second, more time is required to carry out the operation. In this study, we investigated the latter in a consecutive series of patients with PD who underwent DBS of the STN.

\section{Materials and Methods}

This is a prospective cohort study. Twenty-four (14 male) consecutive patients with PD were included. The demographic data of the patients are presented in Table 1. All patients underwent DBS of the STN with MER at the Ondokuz Mayis University (Samsun, Turkey) DBS center and were followed for at least one year postoperatively. Twenty-three patients were operated bilaterally and one patient unilaterally. All patients received preoperative clinical assessments using the Unified Parkinson's Disease Rating Scale (UPDRS) parts I/II/III and IV, Schwab and England and Hoehn \& Yahr scoring in the medication on and off states, and preoperative psychiatric assessments. Inclusion and exclusion criteria for DBS procedures have been described previously ${ }^{13}$. Informed consent was requested and obtained from all patients, and the study was approved by the local medical ethical committee. This study was conducted in accordance with the Declaration of Helsinki.
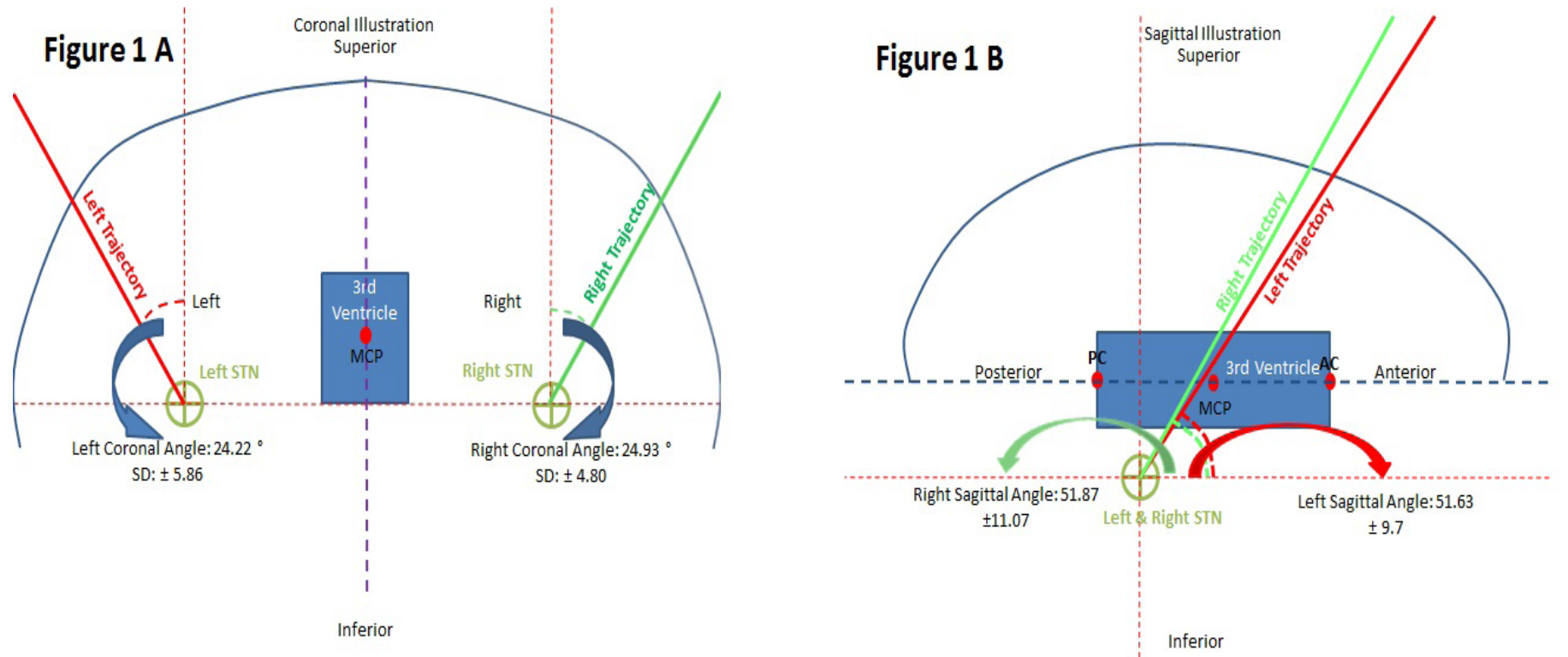

Figure 1: (A) Coronal trajectory angles for the right and left STN trajectories depicted on a coronal illustration. (B) Sagittal trajectory angles for the right and left STN trajectories depicted on a sagittal illustration.

\section{Surgical procedure}

Before surgery and for each patient, a preoperative MRI scan was performed (Siemens, 1.5-Tesla MR Scanner, Erlangen, Germany) and the STN was defined by direct targeting on T2-weighted MR images. The trajectory was planned and the number of MER electrodes was determined by the presence of vessels seen with gadolinium on the T1-weighted MR images. On the morning of surgery, a Leksell G frame was mounted, and stereotaxic computerized tomography (CT) was performed without contrast with a slice thickness of $1 \mathrm{~mm}$ (Aquillon 16 CT scanner, Toshiba, Tokyo, Japan). 
In the next step, the MRI and CT images were fused and stereotactic coordinates were calculated on the work station (Framelink 5, Medtronic Inc. Minneapolis, USA).

The doses of anti-parkinsonian drugs had been reduced in the days before surgery and administration of the drugs was halted $12 \mathrm{~h}$ before the operation. All surgeries were performed under local anesthesia. Following anesthesiologic preparation with dexmedetomidine hydrochloride, a pre-coronal burr hole was made on the contralateral side from the most severe PD symptoms. Before placement of the MER electrodes, the patient's blood pressure was checked. The burr hole was covered with cottonoids to prevent substantial cerebrospinal fluid (CSF) leakage.

MER was performed with polyamide-coated tungsten microelectrodes (Medtronic; microelectrode 291; 10 $\mu \mathrm{m}$ width, impedance $1.1 \pm 0.4 \mathrm{M} \Omega$; measured at $220 \mathrm{~Hz}$ ) in $1 \mathrm{~mm}$ steps from $10 \mathrm{~mm}$ above the target for the first $5 \mathrm{~mm}$, then in $0.5 \mathrm{~mm}$ steps until the end of measured STN activity and the start of substantia nigra pars reticulata (SNr) activity. Signals were recorded with the Leadpoint system (Medtronic, Minneapolis, USA).

Before the test stimulation, which was performed with the same electrodes, patients were examined by the neurologist and baseline values for the cardinal symptoms (rigidity, hypokinesia, and tremor if present) were obtained. The microelectrode with the most typical STN pattern and the longest recordings in mm was always selected for the test stimulation. Intraoperative macrostimulation was performed, with a frequency of $130 \mathrm{~Hz}$ and a pulse width of $60 \mu \mathrm{s}$, in a step-wise fashion every $2 \mathrm{~mm}$ within the recorded STN electrophysiological borders. At each $0.5 \mathrm{~V}$ step, the stimulus intensity was increased until a desired clinical improvement was obtained or a rise in side effects was evaluated by a neurologist. Detailed clinical parameters were scored using the UPDRS including: tremor (if present) and rigidity in all four extremities, finger taps, hand movements and/ or handgrips, and leg agility. If there were positive clinical results with low stimulation parameters, and side effects were absent or only visible at higher amplitudes, the test electrode was removed and replaced with a permanent electrode (Model 3389; Medtronic, Minneapolis, USA). If no satisfactory effect was acquired during the test stimulation, the microelectrode that proved to be the second-best option was chosen for clinical evaluation. This was, typically, the trajectory with the second longest STN activity. The permanent electrode position was verified using fluoroscopy and the remaining microelectrodes were removed. Finally, the permanent electrode was fixed in the burr hole with methyl methacrylate. The same procedure was performed for the contralateral side. After the permanent electrodes' procedures were evaluated and symptomatic bleeding or other structural complications were investigated with T1- and T2-weighted scans, internalization of the pulse generator (Activa PC; Medtronic, Minneapolis, USA) was performed under general anesthesia.

\section{Quantification of time-related details of MER}

The following durations were recorded: the time between the first microelectrode placement as well as the testing of the effects and side effects with macrostimulation, the marking of the chosen trajectory with fluoroscopy, removal of the microelectrode and implantation of macroelectrode, checks on the trajectory with fluoroscopy, and removal of other microelectrodes.

When the calculated and planned trajectories cannot be chosen, a second trajectory is required. The multiple electrodes' advantage is the immediate availability of a second-best trajectory based on the recordings. This approach's advantage is a gain in time.

The average coordinates for the $\mathrm{X}, \mathrm{Y}$, and $\mathrm{Z}$ axes and the average coronal and sagittal trajectory angles were noted for all patients. The number of microelectrodes used on each trajectory for the first and second sides, and the percentage use of the pre-planned trajectory for permanent electrode implantation on the first and second sides, were also calculated. 


\section{Postoperative clinical follow up}

UPDRS part III assessments were scored in the stimulation off/medication off and stimulation on/medication off states. The change in leva-dopa drug dosage (LEDD) at one year postoperatively was evaluated. Early and late postoperative surgical, hardware and psychiatric side effects and complications were investigated.

\section{Statistical analysis}

Data are presented as means \pm standard deviations (SD) and were analyzed using the Wilcoxon signed ranks test. The level of statistical significance was defined as $\mathrm{P}<.05$. All data were analyzed using the SPSS 15.0 software package (Version 15.0).

\section{Results}

The average functional coordinates for the patients who were operated on in the right and/or left sides are presented in Table 2. The average coronal and sagittal trajectory angles are illustrated in Figure 1. One hundred and fifty-nine microelectrodes were used (81 right side, 78 left side) in total for all patients. The average number of microelectrodes used for the right and left sides was $3.5 \pm 0.77$ and $3.4 \pm 0.87$, respectively. The average length of the STN measured with MER, for the first and the second sides to be operated on, were 5.6 $\pm 1.8 \mathrm{~mm}$ and $4.8 \pm 1.5 \mathrm{~mm}$, respectively. Forty-seven permanent electrodes were implanted in all patients (first side: 24, second side: 23). The first side to be operated on included placement of 9 right-side and 15 leftside permanent electrodes; the second side to be operated on included 15 right-side and 8 left-side permanent electrodes (Figure 2).

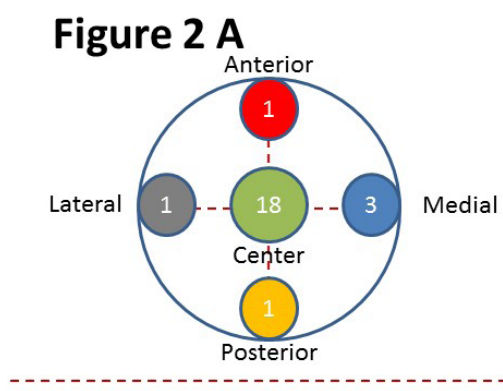

Figure $2 \mathrm{C}$

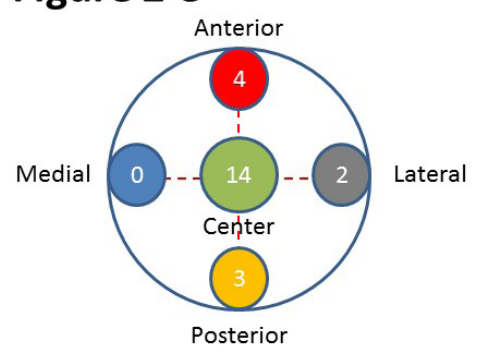

Figure 2 B

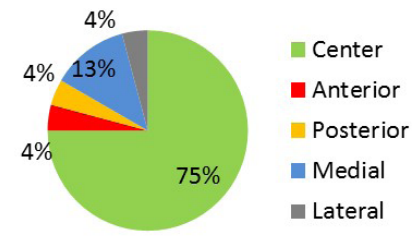

Figure 2 D

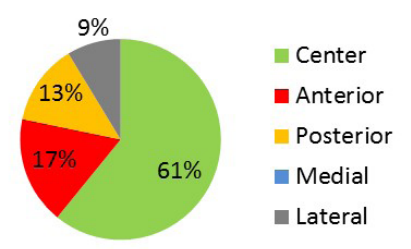

Figure 2:

(A and C) Illustrations of Ben's gun for the procedures on the first side and the second side. The five circles of differing colors within Ben's gun demonstrate the five different trajectories. The numbers shared in these five trajectories denote the number of permanent electrode implantations for each trajectory in this series. The pie charts, shown in panels $\mathrm{B}$ and $\mathrm{D}$, indicate the percentage implantation for each trajectory in Ben's gun for the first side and the second side of the procedure, respectively.

The time spent during the MER phase of the surgery for the first side, second side and total procedure was 23.4 $\pm 6.2 \mathrm{~min} ., 17.4 \pm 6.5 \mathrm{~min}$ and $41.2 \pm 6.3 \mathrm{~min}$, respectively. UPDRS III scores, LEDD in Stim-On and StimOff states at the end of 1 year are presented in Table 1. In this study, there were no intracerebral hemorrhages (ICH) due to radiological assessments. Other intraoperative and postoperative complications, and the side effects related to surgery, hardware, and stimulation, are documented in Table 3. 


\begin{tabular}{lcc}
\hline & $\begin{array}{c}\text { Preoperative } \\
\text { demographic } \\
\text { data of the } \\
\text { patients }\end{array}$ & $\begin{array}{c}\text { Patient } \\
\text { scores at the } \\
\text { end of one } \\
\text { year }\end{array}$ \\
\hline Age & $51.6 \pm 9.63$ & - \\
\hline Disease duration & $10.4 \pm 4.2$ & - \\
\hline LEDD & $1245 \pm 224.5$ & - \\
\hline UPDRS Total Med-off & $82.2 \pm 24.6$ & - \\
\hline UPDRS Total Med-on & $35.1 \pm 9.1$ & - \\
\hline Improvement (\%) & $57.2 \pm 13.8$ & - \\
\hline UPDRS III Med-off & $51.2 \pm 13.2$ & - \\
\hline UPDRS III Med-on & $17.3 \pm 6.1$ & - \\
\hline Improvement (\%) & $66.2 \pm 14.1$ & - \\
\hline H\&Y Med-off & $3.6 \pm 0.6$ & - \\
\hline H\&Y Med-on & $2.5 \pm 0.4$ & - \\
\hline Schwab England \% Med-off & $34 \pm 13.6$ & - \\
\hline Schwab England \% Med-on & $89 \pm 1.8$ & - \\
\hline Beck & $9.1 \pm 2.6$ & - \\
\hline MMSE & $28.6 \pm 0.7$ & - \\
\hline UPDRS III Stim-off Med-off & - & $53.1 \pm 14.4$ \\
\hline UPDRS III Stim-on Med-off & - & $24.3 \pm 15.1$ \\
\hline Improvement (\%) & - & $54.2 \pm 16.8$ \\
\hline LEDD postop & - & $535 \pm 184.5$ \\
\hline Improvement (\%) & & $57 \pm 8.2$ \\
\hline
\end{tabular}

\begin{tabular}{|c|c|c|}
\hline $\begin{array}{l}\text { Complications/ } \\
\text { Side effects }\end{array}$ & $\begin{array}{c}\text { Intraoperative } \\
\text { n (\%) }\end{array}$ & $\begin{array}{c}\text { Postoperative } \\
n(\%)\end{array}$ \\
\hline Intracranial Hemorrhage & - & - \\
\hline Seizure & - & - \\
\hline Mortality & - & - \\
\hline Local Infection & - & $1(4.1)$ \\
\hline Open Wound & - & $1(4.1)$ \\
\hline Presyncope / Syncope & $1(4.1)$ & - \\
\hline Gait Disorders & - & $3(12.5)$ \\
\hline Speech Disorders & $1(4.1)$ & $1(4.1)$ \\
\hline Weight Gain & - & $3(12.5)$ \\
\hline Hypomania & - & $2(8.3)$ \\
\hline Impulse Control Disorders & - & - \\
\hline Pshychotic Disorders & - & - \\
\hline Paresthesia & $3(12.5)$ & - \\
\hline Limitation of Eye Movements & $2(8.3)$ & - \\
\hline Apraxia of Lid Opening & $2(8.3)$ & - \\
\hline Facial Spasm & $1(4.1)$ & - \\
\hline
\end{tabular}

Table 3: Complications and side effects recorded one-year post operation.

Table 1: Patient data prior to the operation and 1-year post operation.

\begin{tabular}{lcc} 
& Right STN & Left STN \\
\hline$X$ & $12.32 \mathrm{~mm} \pm 0.76$ & $-12.24 \mathrm{~mm} \pm 1.04$ \\
\hline$Y$ & $-1.80 \mathrm{~mm} \pm 0.72$ & $-2.31 \mathrm{~mm} \pm 0.74$ \\
\hline$Z$ & $-3.99 \mathrm{~mm} \pm 0.44$ & $-4.17 \mathrm{~mm} \pm 0.39$ \\
\hline
\end{tabular}

Table 2: Average functional coordinates for right and left STN. 


\section{Discussion}

In this study, the average period of time spent during the MER phase of the surgery was $41.2 \pm 6.3$ min for bilateral DBS of the STN. Although there was no significant difference in the average number of microelectrodes used during MER on the first and second sides, there was $\sim 6$ min time difference between procedures on each side. In our experience, the level of cooperation from the patient decreases and confusion sometimes appears during MER of the second side. Accordingly, the surgeons attempt to finalize the MER of the second side as quickly as possible.

As far as we know, there has been no published work specifically measuring the time taken to perform MER during DBS surgeries to date. However, some studies have evaluated the durations of DBS procedures with and without MER ${ }^{7,14-17}$. Kinfe et al. reported that the average total DBS procedure times, calculated with and without MER, are $199 \mathrm{~min}$ in 36 patients and $201 \mathrm{~min}$ in 32 patients, respectively ${ }^{18}$. Interestingly, the report indicates that the time taken for the procedure without MER is longer than when MER is performed. In our experience, MER should start from $10 \mathrm{~mm}$ above the MRI-based target to $5 \mathrm{~mm}$ below it, performed in $1 \mathrm{~mm}$ steps for the first $5 \mathrm{~mm}$, followed by $0.5 \mathrm{~mm}$ steps until cessation of measurable STN activity. About 25 recordings are taken per side. Above the STN, irregular, low-frequency patterns from the anterior thalamus can be recorded, while non-specific patterns can be found in the zona incerta. The STN shows typical electrophysiological activity, consisting of high-voltage spikes, burst firing of cells, and an obvious background expansion. MER is stopped when STN activity disappears and substantia nigra pars reticulata (SNr) activity, which consists of more regular cell firing patterns, appears. One explanation for the burst activity seen in the STN is the loss of dopaminergic input to the STN ${ }^{13,17,18}$. Dopaminergic neurons in the substantia nigra inhibit STN cells. Some publications suggest that the time taken to perform MER may be reduced by starting recordings at $6 \mathrm{~mm}$ instead of $10 \mathrm{~mm}$ from the MRI-based STN target ${ }^{15}$. Although each recording takes 8-10 $\mathrm{s}$, some authors claim even $1 \mathrm{~s}$ of MER per step is sufficient to define the STN's electrophysiological borders ${ }^{16}$. To avoid orientation dysfunction in the patient, we prefer to start MER $10 \mathrm{~mm}$ above the target. We also believe that MER of the STN is an important tool for the identification of the border between the STN and SNr. Even with accurate AP and lateral coordinates, it is not easy to define the most inferior part of the STN if it is difficult to see the border between STN and SNr structures on T2-weighted images. ${ }^{19}{ }^{20}$.

The clinical outcome (improvement of UPDRS III scores) of our series, in which patients were operated on using the MER technique, is similar to results from studies using the same technique in the literature ${ }^{1}$, 10, 21. However, there are many studies performed without MER which have clinical results similar to those using MER 22, 23. Unfortunately, as far as we are aware, there have been no prospective randomized studies comparing clinical outcomes for patients who have undergone DBS operations with and without MER. Reck et al. published a series comparing the clinical outcomes of 32 patients with PD who underwent DBS of the STN, including 22 patients who were operated on with MER, and 10 patients without MER ${ }^{12}$. They found a significantly better clinical outcome (UPDRS III scores) in patients undergoing MER compared with those who did not undergo MER.

In our study, there was no ICH occurrence, which is the most feared complication of DBS in the STN using MER. The estimated risk of an ICH in DBS of the STN varies between $0.2 \%$ and $5.6 \%$ in the literature ${ }^{10,22,24,}$ ${ }^{25}$. Hariz et al. claim that non-MER guided DBS surgery is five times safer in comparison to MER guided DBS surgeries in terms of ICH risk ${ }^{26}$. Xiaowu et al. reported that increasing microelectrode trajectories seemed to increase the risk of $\mathrm{ICH}$, but no statistically significant difference was found ${ }^{23}$. In our previous study, which included 220 patients with PD who underwent DBS of the STN, we found that the overall calculated risk of ICH was $1.81 \%$ per patient, $0.3 \%$ per recording electrode, and $0.23 \%$ per brain insertion ${ }^{11}$. Other complications involved with performing DBS of the STN found in this study are similar to those reported in other publications ${ }^{27-30}$. 
In this study, the percentage use of central trajectories for permanent electrode implantations in the first and second sides were $75 \%$ and $61 \%$, respectively. Although we used cottonoids to prevent brain shift due to CSF leakage, we believe that the percentage difference in use of the central trajectory for the first and second sides is related to this factor. Therefore, the MER of the STN may be much more important for electrode implantation on the second side. With the use of MER, error related to CSF leakage could be minimized or eliminated. Bour et al. reported that, in $50 \%$ of their cases, the central trajectory was used for permanent electrode implantation in a study of 29 PD patients when the authors performed DBS of the STN ${ }^{15}$. In their series, they used a combination of direct and indirect methods on the work station for preoperative targeting. In another study, Reck et al. calculated the central trajectory implantation rate to be $73 \%$ in 22 patients with PD when DBS of the STN was performed ${ }^{12}$. In our previous study, we reported a central trajectory implantation rate of $\sim 70 \%$ using a combination of direct and indirect targeting methods ${ }^{31}$. Toda et al. reported that the implant rate of permanent DBS electrodes to the central trajectory was $81 \%$ in their series in which a $3 \mathrm{~T}$ MRI machine was used. Even the transition from $1.5 \mathrm{~T}$ to $3.0 \mathrm{~T}$ seems to improve targeting accuracy. A non-central trajectory is currently used for one in five patients ${ }^{14}$.

Today, it is well-accepted that, through advancements in MRI techniques and technologies, it is feasible to perform the DBS procedure without using MER. However, we still believe that MER offers the DBS surgical team a choice of using a second trajectory, when needed, to improve the effect of the therapy and reduce the incidence of side effects. 


\section{References}

1. Deuschl G, Schade-Brittinger C, Krack P, et al. A randomized trial of deep-brain stimulation for Parkinson's disease. N Engl J Med. 2006;355(9):896-908.

2. Odekerken VJ, van Laar T, Staal MJ, et al. Subthalamic nucleus versus globus pallidus bilateral deep brain stimulation for advanced Parkinson's disease (NSTAPS study): a randomised controlled trial. The Lancet Neurology. 2013;12(1):37-44.

3. Janssen ML, Duits AA, Tourai AM, et al. Subthalamic nucleus high-frequency stimulation for advanced Parkinson's disease: motor and neuropsychological outcome after 10 years. Stereotact Funct Neurosurg. 2014;92(6):381-7.

4. Tonge M, Kocabicak E, Ackermans L, Kuijf M, Temel Y. Final electrode position in subthalamic nucleus deep brain stimulation surgery: a comparison of indirect and direct targeting methods. Turk Neurosurg. 2016;10:1019-5149.

5. Benabid AL, Pollak P, Gao D, et al. Chronic electrical stimulation of the ventralis intermedius nucleus of the thalamus as a treatment of movement disorders. J Neurosurg. 1996;84(2):203-14.

6. Starr PA. Placement of deep brain stimulators into the subthalamic nucleus or globus pallidus internus: technical approach. Stereotact Funct Neurosurg. 2002;79(3-4):118-45.

7. McClelland S. A cost analysis of intraoperative microelectrode recording during subthalamic stimulation for Parkinson's disease. Mov Disord. 2011;26(8):1422-7.

8. Savas A, Bozkurt M, Akbostanc1 C. A comparison between stereotactic targeting methods of the subthalamic nucleus in cases with Parkinson's disease. Stereotactic and Functional Neurosurgery: Springer; 2013. p. 35-41.

9. Hariz MI. Complications of deep brain stimulation surgery. Mov Disord. 2002;17(S3).

10. Temel Y, Wilbrink P, Duits A, et al. Single electrode and multiple electrode guided electrical stimulation of the subthalamic nucleus in advanced Parkinson's disease. Operative Neurosurgery. 2007;61(suppl_5):ONS346-ONS57.

11. Tonge M, Ackermans L, Kocabicak E, et al. A detailed analysis of intracerebral hemorrhages in DBS surgeries. Clin Neurol Neurosurg. 2015;139:183-7.

12. Reck C, Maarouf M, Wojtecki L, et al. Clinical outcome of subthalamic stimulation in Parkinson's disease is improved by intraoperative multiple trajectories microelectrode recording. Journal of Neurological Surgery Part A: Central European Neurosurgery. 2012;73(06):377-86.

13. Kocabicak E, Temel Y. Deep brain stimulation of the subthalamic nucleus in Parkinson's disease: surgical technique, tips, tricks and complications. Clin Neurol Neurosurg. 2013;115(11):2318-23.

14. Toda H, Sawamoto N, Hanakawa T, et al. A novel composite targeting method using high-field magnetic resonance imaging for subthalamic nucleus deep brain stimulation. Journal of neurosurgery. 2009;111(4):737-45.

15. Bour LJ, Contarino MF, Foncke EM, et al. Long-term experience with intraoperative microrecording during DBS neurosurgery in STN and GPi. Acta neurochirurgica. 2010;152(12):2069-77.

16. Shamir RR, Zaidel A, Joskowicz L, Bergman H, Israel Z. Microelectrode recording duration and spatial density constraints for automatic targeting of the subthalamic nucleus. Stereotact Funct Neurosurg. 2012;90(5):325-34.

17. Zeiler F, Wilkinson M, Krcek J. Subthalamic nucleus deep brain stimulation: an invaluable role for MER. Canadian journal of neurological sciences. 2013;40(4):572-5.

18. Kinfe TM, Vesper J. The impact of multichannel microelectrode recording (MER) in deep brain stimulation of the basal ganglia. Stereotactic and functional neurosurgery: Springer; 2013. p. 27-33.

19. Schlaier JR, Habermeyer C, Warnat J, et al. Discrepancies between the MRI-and the electrophysiologically defined subthalamic nucleus. Acta neurochirurgica. 2011;153(12):2307-18.

20. Zhu X, Hamel W, Schrader B, et al. Magnetic resonance imaging-based morphometry and landmark correlation of basal ganglia nuclei. Acta Neurochir (Wien). 2002;144(10):959-69.

21. Kleiner-Fisman G, Herzog J, Fisman DN, et al. Subthalamic nucleus deep brain stimulation: Summary and meta-analysis of outcomes. Mov Disord. 2006;21(S14).

22. Ben-Haim S, Asaad WF, Gale JT, Eskandar EN. Risk factors for hemorrhage during microelectrode-guided deep brain stimulation and the introduction of an improved microelectrode design. Neurosurgery. 2009;64(4):754-63.

23. Xiaowu H, Xiufeng J, Xiaoping Z, et al. Risks of intracranial hemorrhage in patients with Parkinson's disease receiving deep brain stimulation and ablation. Parkinsonism Relat Disord. 2010;16(2):96-100. 
24. Binder DK, Rau G, Starr PA. Hemorrhagic complications of microelectrode-guided deep brain stimulation. Stereotact Funct Neurosurg. 2003;80(1-4):28-31.

25. Voges J, Waerzeggers Y, Maarouf M, et al. Deep-brain stimulation: long-term analysis of complications caused by hardware and surgery—experiences from a single centre. J Neurol Neurosurg Psychiatry. 2006;77(7):868-72.

26. Hariz MI. Safety and risk of microelectrode recording in surgery for movement disorders. Stereotact Funct Neurosurg. 2002;78(3-4):146-57.

27. Beric A, Kelly PJ, Rezai A, et al. Complications of deep brain stimulation surgery. Stereotact Funct Neurosurg. $2001 ; 77(1-4): 73-8$.

28. Lyons KE, Wilkinson SB, Overman J, Pahwa R. Surgical and hardware complications of subthalamic stimulation A series of 160 procedures. Neurology. 2004;63(4):612-6.

29. Blomstedt P, Hariz M. Hardware-related complications of deep brain stimulation: a ten year experience. Acta Neurochir (Wien). 2005;147(10):1061-4.

30. Doshi PK. Long-term surgical and hardware-related complications of deep brain stimulation. Stereotact Funct Neurosurg. 2011;89(2):89-95.

31. Kocabıcak E AD, Ozaydın I, Jahanshahı A, K. H Tan S, Onar M, Boke O, Kurt M, Guz H, Terzı M, Alptekın O, Temel Y. Does Probe's Eye Subthalamic nucleus length on T2W MRI Correspond with Microelectrode Recording in Patients with Deep Brain Stimulation for Advanced Parkinson's Disease? Turk Neurosurg. 2013;23(5):658-65. 
Chapter 7

Discussion and Conclusion 


\section{Discussion}

In this study, the methodological aspects of DBS (Deep Brain Stimulation) surgery technique were reported, investigated and questioned. Stereotactic frames, stereotactic localizers, stereotactic planning stations, radiological imaging systems, neurophysiological recording systems, pros and cons of these technologies were reported and discussed through five different studies. In addition to the retrospective qualitative analysis of experienced technical events and complications, potential scenarios were designed and the effects of these scenarios on surgical instruments and systems were investigated. Moreover, pros and cons of MER (Microelectrode Recording) methodology in DBS surgeries were discussed and presented with respect to our perspective and approach to DBS therapy.

The neurophysiological aspects of DBS surgery are still highly debated in the scientific community. Safety, necessity and pros and cons of MER systems used in the examination of neurophysiological signals are among the issues that are frequently discussed in the literature ${ }^{1-9}$. However, we have seen that there are very limited data in the literature regarding to the complications that may be encountered during the use of the instruments and technologies for DBS surgeries and management strategies of these complications. With this detailed work focusing on methodological aspects of DBS surgeries, we have not only added our own experience and comments to the existing discussions but also provided a new perspective and contribution to the literature.

With respect to our analyses, the technical complications were mostly originated from stereotactic frames and stereotactic planning stations (Chapter 2). In prior studies related to the stereotactic surgery method, the focus was mostly on stereotactic accuracy comparison of individual stereotactic surgery systems and stereotactic surgery methodologies ${ }^{10-12}$. Nevertheless, in a very limited number of studies, the pitfalls in stereotactic surgeries were highlighted and at some point, these studies mentioned similar complications in comparison to ours. Zrinzo et. Al. emphasized the importance of stereotactic frame and planning station-related issues such as the mounting of stereotactic frame to the patient head, patient positioning in the operating room, importance of stereotactic equipment maintenance and image fusion process quality ${ }^{13}$. In the study we presented, the issues highlighted by Zrinzo et. Al were detailed with more specific cases which were experienced in our DBS centers. Additionally, complications related to stereotactic localizers which we do not encounter in the literature were presented with different aspects. Particularly, complications experienced during the integration of stereotactic localizers with planning stations, stereotactic frames and radiological imaging systems were detailed and introduced within this study.

In the clinical application of DBS surgery, technical complications are not only linked with stereotactic frames and planning stations. Complications arising from electrophysiological recording systems may lead planned DBS cases to be delayed or canceled or to be performed with a different surgical technique. Artifacts that may prevent the acquisition of neurophysiological recordings, recording problems related to recording electrodes, and strategies to deal with these problems seem to be important need in the literature. Studies that focus on this direction will add different dimensions to the discussion of methodological aspects of DBS surgeries. However, this topic was not covered by the present study. 
Stereotactic accuracy is another important topic that needs to be questioned from different perspectives within the discussion of methodological aspects of DBS surgeries. With this purpose we designed a phantom model in order to investigate the effect of asymmetrical frame mounting on stereotactic accuracy (Chapter 3). Three individual stereotactic frame mounting scenarios were designed with this purpose. We examined and compared the final coordinates of the pre-defined target points for each scenario on three individual stereotactic planning software packages. Then, we calculated the stereotactic coordinates manually by two individual observes for these three scenarios and included the results into the comparison. As a result of the study, we showed that asymmetric stereotactic frame mounting can lead to inaccuracies for the $\mathrm{Z}$ coordinate, particularly when manual calculation method is considered.

There are several studies in the literature focusing on the accuracy comparison of individual stereotactic surgery systems and methodologies within each other. In a very limited number of studies, the accuracy comparison between stereotactic planning software packages and manual calculation method was documented ${ }^{10-12}$. The main purpose of this study was to investigate the effect of the asymmetrical frame mounting on software and manual calculation methods; however, we presented an accuracy comparison of the most frequently and recently used stereotactic planning software packages and the manual calculation method.

In Chapter 4, we investigated the effect of computed tomography (CT) slice thickness parameter on stereotactic calculations with another phantom study. First, we obtained seven CT image series of the DBS electrode implanted phantom with different slice thicknesses. Then, we merged these CT series with thin sliced MR images of the same phantom using a stereotactic planning station and software. Within the phantom, we identified a specific recognizable part of the implanted DBS electrode as the target point for all merged series. Finally, we compared the computed/calculated stereotactic coordinates of the specified target from all merged series. We found that the stereotactic calculations were not affected by the changes in CT slice thickness parameter (between $0.5 \mathrm{~mm}$ and $5 \mathrm{~mm}$ ) when a $2 \mathrm{~mm}$ MRI was used as co-registration. Apart from all these, it would be valuable for the literature to consider the other CT parameters as an investigation area that would affect stereotactic calculations.

Another focus area within the study was the microelectrode recording (MER) method which has been causing controversies in neurosurgical community. Firstly (Chapter 5), we reviewed the literature and focused on how MER methodology started to be used in clinical applications historically. Then, the necessity of MER methodology after the technological developments in MR imaging in the literature was discussed with current controversies.

In the literature, important studies were supporting the fact that STN targeting performed on T2 weighted MR images was highly correlated with the anatomic locations determined with MER and macrostimulation methods $s^{14-16}$. Moreover, the results of our previous study which was investigating the correlation between radiological and neurophysiological borders of STN was in line with literature ${ }^{17}$. However, in one third of the DBS electrode implantations performed in our centers, the central trajectory of the MER system was not chosen for the permanent electrode implantation ${ }^{18}$. Consequently, we emphasized that intraoperative electrophysiology is not indispensable for DBS surgeries; however, it is important that the MER methodology and technology provides the chance to correct systemic errors that may arise during the application of stereotactic surgical approach. 
Finally in Chapter 6, we focused another controversial topic on the use of MER with a prospective study which is the operational time of MER method. We found in the literature that one of the reasons why MER method is questioned by some centers is the incremental effect of the method on operational time ${ }^{6,19-21}$. With that, we investigated the average time spent for MER during PD DBS surgeries with a prospective study involving twenty-four patients who were operated in our centers. MER usage durations for the first operated side of the brain, for the second operated side of the brain and the complete operation were $23.4 \pm 6.2$ minutes, $17.4 \pm 6.5$ minutes and $41.2 \pm 6.3$ minutes respectively.

As we compared the results of our study with previous ones in the literature, we were convinced that MER procedure had an incremental impact on the operational time of the surgery. However, despite its disadvantages in terms of operational time, we emphasized MER's superiority in determining the borders of the target and relevant anatomical structures with sub-millimeter precision when compared to MR guided implantation technique. In addition, we calculated the percentage of central trajectory choice for the permanent electrode implantation as $75 \%$ for the first side and $61 \%$ for the second side for a bilateral PD STN DBS surgery within the same series. As a result, we emphasized that MER procedure may be more valuable for the permanent electrode implantation of second side of a bilateral DBS surgery than the first side in order to compensate error sources that might arise intraoperatively.

As we mentioned in previous sections, stereotactic surgery is an application that many factors contribute to the success of the application such as intraoperative factors, human hand sensitivity, surgical instrument's physical conditions, surgical instrument usage and integration of individual stereotactic surgery technologies between each other. The excessive number of factors involving in the success of stereotactic surgery approach may also increase the number of error sources that may have effect on stereotactic accuracy. In this sense, we believe that MER method provides an important maneuverability for DBS surgeries in order to compensate possible error sources intraoperatively and is still preferred in many centers despite the use of high-resolution radiological imaging technologies.

\section{Conclusion}

Modern surgical instruments, technologies and radiological imaging modalities are used extensively in the clinical application of DBS surgery. At this point where technology and surgery intersect, it is inevitable to experience various technical complications during the integration and use of individual technologies. Identification and management of such complication sources is critical to the success of DBS therapy.

Asymmetric stereotactic frame mounting which appears as a technical complication source that might lead errors on stereotactic accuracy was investigated with a phantom study and we found that asymmetric mounting of stereotactic frame can lead inaccuracies in superoinferior axis ( $Z$ coordinate) if manual calculation method is considered for use. In addition, the effect of stereotactic CT slice thickness increase on stereotactic calculations was investigated with another phantom study and no effect was observed when CT images were fused with thin slice MR images.

The use, advantages, disadvantages and necessity of MER methodology and technologies in DBS surgeries are still being discussed today. MER method has an incremental effect on the operational time of DBS surgeries. However, MER provides an opportunity of correcting systemic errors that may arise during DBS surgeries. For the STN DBS surgeries performed bilaterally, the permanent lead implantation rate to the central trajectory for the second operated STN is lower than the first operated side. In this sense, MER could be worthwhile to compensate the systemic errors specifically for the second operated STN in a bilateral DBS surgery. 


\section{References}

1. Moro E. Neurosurgery: Complications of DBS surgery—insights from large databases. Nature Reviews Neurology. 2016;12(11):617.

2. Fenoy AJ, Simpson RK. Risks of common complications in deep brain stimulation surgery: management and avoidance. Journal of neurosurgery. 2014;120(1):132-9.

3. Xiaowu H, Xiufeng J, Xiaoping Z, et al. Risks of intracranial hemorrhage in patients with Parkinson's disease receiving deep brain stimulation and ablation. Parkinsonism \& related disorders. 2010;16(2):96-100.

4. Hariz MI. Safety and risk of microelectrode recording in surgery for movement disorders. Stereotactic and functional neurosurgery. 2002;78(3-4):146-57.

5. Kinfe TM, Vesper J. The impact of multichannel microelectrode recording (MER) in deep brain stimulation of the basal ganglia. Stereotactic and functional neurosurgery: Springer; 2013. p. 27-33.

6. McClelland III S. A cost analysis of intraoperative microelectrode recording during subthalamic stimulation for Parkinson's disease. Movement disorders. 2011;26(8):1422-7.

7. Bour LJ, Contarino MF, Foncke EM, et al. Long-term experience with intraoperative microrecording during DBS neurosurgery in STN and GPi. Acta neurochirurgica. 2010;152(12):2069-77.

8. Shamir RR, Zaidel A, Joskowicz L, Bergman H, Israel Z. Microelectrode recording duration and spatial density constraints for automatic targeting of the subthalamic nucleus. Stereotactic and functional neurosurgery. 2012;90(5):325-34.

9. Zeiler F, Wilkinson M, Krcek J. Subthalamic nucleus deep brain stimulation: an invaluable role for MER. Canadian journal of neurological sciences. 2013;40(4):572-5.

10. Bot M, Van Den Munckhof P, Bakay R, Sierens D, Stebbins G, Metman LV. Analysis of stereotactic accuracy in patients undergoing deep brain stimulation using Nexframe and the Leksell frame. Stereotactic and functional neurosurgery. 2015;93(5):316-25.

11. Sharma M, Rhiew R, Deogaonkar M, Rezai A, Boulis N. Accuracy and precision of targeting using frameless stereotactic system in deep brain stimulator implantation surgery. Neurology India. 2014;62(5):503.

12. Slavin KV, Anderson GJ, Burchiel KJ. Comparison of three techniques for calculation of target coordinates in functional stereotactic procedures. Stereotactic and functional neurosurgery. 1999;72(2-4):192-5.

13. Zrinzo L. Pitfalls in precision stereotactic surgery. Surgical neurology international. 2012;3(Suppl 1):S53.

14. Bejjani B-P, Dormont D, Pidoux B, et al. Bilateral subthalamic stimulation for Parkinson's disease by using threedimensional stereotactic magnetic resonance imaging and electrophysiological guidance. Journal of neurosurgery. 2000;92(4):615-25.

15. Egidi M, Rampini P, Locatelli M, et al. Visualisation of the subthalamic nucleus: a multiple sequential image fusion (MuSIF) technique for direct stereotaxic localisation and postoperative control. Neurological Sciences. 2002;23(2):s71-s2.

16. Starr PA, Christine CW, Theodosopoulos PV, et al. Implantation of deep brain stimulators into subthalmic nucleus: technical approach and magnetic imaging_-verified electrode locations. Journal of neurosurgery. 2002;97(2):370-87.

17. Kocabıcak E, Aygun D, Ozaydın I, et al. Does probe's eye subthalamic nucleus length on T2W MRI correspond with microelectrode recording in patients with deep brain stimulation for advanced Parkinson's disease? Turkish neurosurgery. 2013;23(5):658-65.

18. Tonge M, Kocabıçak E, Ackermans L, Kuijf M, Temel Y. Final electrode position in subthalamic nucleus deep brain stimulation surgery: a comparison of indirect and direct targeting methods. 2016.

19. Reck C, Maarouf M, Wojtecki L, et al. Clinical outcome of subthalamic stimulation in Parkinson's disease is improved by intraoperative multiple trajectories microelectrode recording. Journal of Neurological Surgery Part A: Central European Neurosurgery. 2012;73(06):377-86.

20. Savas A, Bozkurt M, Akbostanc1 C. A comparison between stereotactic targeting methods of the subthalamic nucleus in cases with Parkinson's disease. Stereotactic and Functional Neurosurgery: Springer; 2013. p. 35-41.

21. Temel Y, Wilbrink P, Duits A, et al. Single electrode and multiple electrode guided electrical stimulation of the subthalamic nucleus in advanced Parkinson's disease. Operative Neurosurgery. 2007;61(suppl_5):ONS346-ONS57. 
Summary 

In this thesis, the methodological aspects of DBS (Deep Brain Stimulation) surgery technique were reported, investigated and questioned by 5 studies. In Chapter 2, we documented technical events encountered during DBS surgeries from 1999 onwards with a retrospective qualitative analysis. The technical complications were mostly originated from stereotactic frames and stereotactic planning stations. Stereotactic frame related complications included movement-related fixation problems, head anatomy related problems, and lack of maintenance related issues. Moreover, we detected some localizer related complications which were compatibility issues of the stereotactic localizer and planning station, field of view effect on fiducials, air bubbles in localizers using liquid solutions, and disengaged localizer effect. Lastly, planning station related complications included image fusion failures and CSF signal effect on image fusion.

Frame mounting is considered one of the most critical steps in stereotactic DBS surgeries. In this sense, we addressed the question whether an asymmetrically mounted frame influences the accuracy of stereotactic electrode implantation (Chapter 3). After magnetic resonance imaging (MRI) scan of a citrullus lanatus, symmetric and asymmetric mounting of the frame, which could occur in clinical scenarios, was performed with computed tomography (CT). We analyzed these images with tree different stereotactic planning stations. Our results showed that an asymmetrically mounted frame did not affect the accuracy in the mediolateral axis (X-coordinate) or the anteroposterior axis (Y-coordinate). However, we found a clinically relevant error in the superoinferior axis (Z-coordinate). These results suggested that asymmetrical frame mounting can lead to stereotactic inaccuracy in the superoinferior axis (Z-coordinate).

The slice thickness of a stereotactic CT is a matter of discussion but often thin slices are used and recommended because of the high reported accuracy. In Chapter 4 we investigated if the stereotactic CT slice thickness has an effect on stereotactic calculations and its accuracy. We used a phantom, a citrullus lanatus, implanted with a DBS electrode. The deepest contact point of the electrode was the defined fixed target. Stereotactic coordinates, ring and arc angles from all stereotactic CT scans with different slice thicknesses were recorded and compared after fusion with a thin sliced non-stereotactic MRI. We found no significant effect of increasing slice thickness of CT on the stereotactic calculation when fused with a thin-sliced MRI in a simulated stereotactic DBS setting.

Another focus area within the study was the microelectrode recording (MER) method. Firstly (Chapter 5), we reviewed the literature and focused on how MER methodology started to be used in clinical applications historically. Then, the necessity of MER methodology after the technological developments in MR imaging in the literature was discussed with current controversies. Finally, we emphasized that intraoperative electrophysiology is not indispensable for DBS surgeries; however, it is important that the MER methodology and technology provides the chance to correct systemic errors that may arise during the application of stereotactic surgical approach.

In Chapter 6, we focused on operational time of MER method. We investigated the average time spent for MER during Parkinson's Disease (PD) DBS surgeries with a prospective study. MER usage durations for the first operated side of the brain, for the second operated side of the brain and the complete operation were recorded and calculated respectively. In addition, we calculated the percentage of central trajectory choice for the permanent electrode implantation for the first side and for the second side for a bilateral PD STN DBS surgery within the same series. The results showed that MER procedure has an incremental impact on the operational time of the surgery. However, we concluded that MER method provides an important maneuverability for DBS surgeries in order to compensate possible error sources intraoperatively. 

Impact 

Deep brain stimulation (DBS) is a surgical therapy which has been applied to more than 160,000 patients globally with neurological and psychiatric disorders since the first clinical application and it has been accepted as the standard of care for refractory motor circuit disorders ${ }^{1}$. The annual rate of increase in the number of cases in humanitarian device exempt (HDE) or emerging indications and Food Drug Administration (FDA) approved indications is $36.1 \%$ and $7 \%$, respectively ${ }^{2}$. Between 1991-2014, over 7000 scientific papers were published about $\mathrm{DBS}^{3}$. The clinical application of DBS therapy stands out as an important area that draws the attention of scientists and it is described as one of the most important developments in the field of clinical neuroscience in the last two decades.

Parkinson's disease and Epilepsy are the indications with the highest prevalence in the general population among the FDA approved indications of DBS therapy ${ }^{4}$. Parkinson's disease is the most common movement disorder disease with a prevalence of $0.3 \%$ in the general population and $1 \%$ over the age of 60 . It is a heavier burden in aging populations ${ }^{5}$. Epilepsy is seen in approximately 50 million patients around the world while $20 \%$ of these patients have resistance to anti-epileptic medications ${ }^{6}$. Today, deep brain stimulation therapy is a proven and promising option for patients suffering from these high prevalence neurological disorders ${ }^{7}$.

One of the factors affecting the success rate of DBS therapy is the anatomical positioning of the implantable products to the desired structures in the brain with high accuracy. Despite the use of high technology surgical systems and instruments for this purpose, complications linked to several sources are frequently reported in the literature. In a study conducted in the United States between 2004 and 2013, DBS surgeries were investigated from Medicare and Medicaid Services (CMS) and the National Surgical Quality Improvement Program (NSQIP) and the ratio of DBS electrode revision or electrode removal operations to total DBS surgeries was found $15.2 \%$ in the first system and $34 \%$ in the second system respectively ${ }^{8}$. Moreover, revision cases due to improper targeting or therapeutic efficacy problems were $48 \%$ of total electrode revision cases ${ }^{8}$. However, there is no clear data about the reason behind these improper targeted first electrode implantations resulting with lead revision procedures. In addition, there is no study about the financial burden of electrode revision surgeries on health care systems.

DBS electrode revision surgeries can bring time loss and incremental costs to healthcare systems. According to a study in US, an average DBS electrode removal operation and an average re-implantation duration was measured $76.38 \mathrm{~min}(\mathrm{SD} \pm 43.10)$ and $256.50 \mathrm{~min}(\mathrm{SD} \pm 166.68)$ respectively ${ }^{9}$. In another study conducted in the US, the average cost of a DBS operation and the average cost of managing complications following the first surgery was reported $\$ 40,063$ and $\$ 4,665.10$ respectively ${ }^{10}$.

Deep brain stimulation surgeries are mostly performed under two common implantation methods which are asleep and awake surgeries ${ }^{11}$. Awake DBS surgeries are preferred due to the physiological conditions required for microelectrode recording (MER) and macrostimulation applications, while these applications are generally not used in asleep surgeries. However, these applications can bring additional burdens to the centers in terms of time and cost. Moreover, there are opinions that these applications increase the surgical risks of DBS surgeries ${ }^{12}$. However, despite all the time losses and additional costs, the benefits of MER and macrostimulation applications are still supported and advocated by scientists ${ }^{13}$. 
In this study, we reported the technical complications experienced in our centers that could lead improper surgical planning, misplaced electrode implantations, additional surgical interventions, patient dissatisfactions, time losses and additional costs which could add extra burden to healthcare systems and providers. Following this, we simulated usage patterns that could lead adverse events described above and investigated to what extent these usage patterns affect the accuracy and the output results of surgical planning systems and instruments. Moreover, we discussed the pros and cons of the MER application, which is an important discussion area for DBS literature. We investigated the time-wise effect of MER application for DBS surgeries and shared our perspective on the benefits it provides. Over all, this study will present a different perspective to the DBS literature on management and prevention of adverse events related to technical aspects of DBS surgeries, patient dissatisfactions, loss of time and incremental costs. 


\section{References}

1. Lozano AM, Lipsman N, Bergman H, et al. Deep brain stimulation: current challenges and future directions. Nature Reviews Neurology. 2019;15(3):148-60.

2. Youngerman BE, Chan AK, Mikell CB, McKhann GM, Sheth SA. A decade of emerging indications: deep brain stimulation in the United States. Journal of neurosurgery. 2016;125(2):461-71.

3. Ineichen C, Christen M. Analyzing 7000 texts on deep brain stimulation: what do they tell us? Frontiers in integrative neuroscience. 2015;9:52.

4. Pereira EA, Green AL, Nandi D, Aziz TZ. Deep brain stimulation: indications and evidence. Expert review of medical devices. 2007;4(5):591-603.

5. Lew M. Overview of Parkinson's disease. Pharmacotherapy: The Journal of Human Pharmacology and Drug Therapy. 2007;27(12P2):155S-60S.

6. Li MC, Cook MJ. Deep brain stimulation for drug-resistant epilepsy. Epilepsia. 2018;59(2):273-90.

7. Fisher R, Salanova V, Witt T, et al. Electrical stimulation of the anterior nucleus of thalamus for treatment of refractory epilepsy. Epilepsia. 2010;51(5):899-908.

8. Rolston JD, Englot DJ, Starr PA, Larson PS. An unexpectedly high rate of revisions and removals in deep brain stimulation surgery: analysis of multiple databases. Parkinsonism \& related disorders. 2016;33:72-7.

9. Frizon LA, Nagel SJ, May FJ, et al. Outcomes following deep brain stimulation lead revision or reimplantation for Parkinson's disease. Journal of neurosurgery. 2018;130(6):1841-6.

10. Stroupe KT, Smith B, Weaver FM, et al. Healthcare Utilization and Costs for Patients With Parkinson's Disease After Deep Brain Stimulation. Movement disorders clinical practice. 2019;6(5):369-78.

11. Wang J, Ponce FA, Tao J, et al. Comparison of awake and asleep deep brain stimulation for Parkinson's disease: a detailed analysis through literature review. Neuromodulation: Technology at the Neural Interface. 2020;23(4):444-50.

12. Hariz MI. Safety and risk of microelectrode recording in surgery for movement disorders. Stereotactic and functional neurosurgery. 2002;78(3-4):146-57.

13. Bjerknes S, Toft M, Konglund AE, et al. Multiple microelectrode recordings in STN-DBS surgery for Parkinson's disease: a randomized study. Movement Disorders Clinical Practice. 2018;5(3):296-305. 

Acknowledgements 



\section{Dr. Yasin Temel}

I would like to express my sincere gratitude to Prof. Yasin Temel, my supervisor, who encouraged me and gave me the opportunity to start my PhD studies at Maastricht University. He has been a wise guide, true supporter and passionate motivator at every single stage of this $\mathrm{PhD}$ adventure. He shared his expertise for creation of the scientific content of this study at every opportunity he could find, from laboratory environments to airports. He and his family hosted me during my visits to Maastricht with compassion. He has always been an important career coach to me with his perspective to neuromodulation and medical device industries. The brainstorming sessions we had during our countless trips to different locations of the country with medical education purpose on DBS therapy built the background of this thesis. It was always a great pleasure to have the chance to meet him and feel his support at every single stage.

\section{Dr. Ersoy Kocabiçak}

He valued my potential and professional career greatly and has always been one of my greatest sources of inspiration to start my $\mathrm{PhD}$ studies. I felt his endless support for promoting my professional experience with an academic title at every moment from the beginning. Looking back to the dozens of scientific and professional projects designed and completed, I'm proud of the things we have brought out with his collaboration and guidance.

\section{Dr. Felix Gubler}

He is one of the scientists who had important role in the emergence of this study. He contributed to this work in the design, application, measurement, evaluation and article writing stages of the experiments and supported my studies during all my visits to Maastricht University. The scientific partnership and friendship we built will always be precious and remembered.

\section{Dr. Janardan Vaidyanathan}

I also would like to express my gratitude to my first DBS teacher, Dr. Janardan Vaidyanathan, who sowed the first seeds of my interest in DBS therapy. He made a great effort on me to teach the therapy in many different aspects from radiology to neurophysiology. We supported dozens of DBS cases in different parts of the country, traveled for countless hours and shared our destiny on the road. I'm proud of being a companion to him during one single step of his countless trips to deliver solutions to people who are miles away from his home.

\section{Dr. Majed Aldehri}

I would also like to thank Dr. Majed Aldehri for his sincerity and companionship, who took care of me personally and tried to involve me to the social life during my time in Maastricht. Your kindness and helpfulness will always be remembered. 


\section{Ülker Alptekin, Tahsin Alptekin, Arda Alptekin}

I would also like to share my endless gratitude to my beloved mother and father, Ülker and Tahsin Alptekin, who raised and brought me to these days, put their lives in the second plan for my education and made all kinds of sacrifice without hesitation. Any success I achieved in my life made and will make you both happier and prouder than anyone else. Special thanks are due to my old roommate, home mate, gaming friend and brother, Arda Alptekin, for his companionship under the same roof starting from my hometown, Izmit days.

\section{Didem Savaş Alptekin}

I would like to express my special thanks to my lovely wife Didem Savaş Alptekin who touched my life in a hilarious way, and made me look at the world from a different perspective. She joined my journey almost at the same period that I started my $\mathrm{PhD}$ adventure and encouraged me with all her support at every single moment of this challenge. I'm sure that we will achieve much better things together. This is the beginning.

I'm also very thankful to my 10 years' manager and colleague, Mrs. Dilek Çağlar, who involved me to her team in 2010 and introduced me to neuromodulation therapies for the first time in my life which was a real turning point for my career.

I would also like to express my gratitude to Maastricht University School for Mental Health and Neuroscience for giving me the opportunity and the academic platform to perform all these studies. I will be carrying the banner of Maastricht University with honor for a lifetime. I would also like to thank Maastricht University Medical Center for their permission to use all their basis and medical equipment.

I would also like to express my deepest gratitude to the assessment committee assigned for the evaluation of the scientific content of this thesis. The oral defense decision of scientific content will always be an honor to me.

Last but not least, I am also thankful to my lifetime friend, Mr. Çağlar Kaya, who shared his expertise and supported me for the proof readings of English texts. Finally, I would like thank to all of my friends who touched my life sometime, somehow. All the butterfly effects will be appreciated and remembered. 


Curriculum Vitae 

Onur Alptekin was born in Ankara in 1984. After completing his primary and high school education in Kocaeli/ Turkey, he returned to Ankara in 2002 for his bachelor degree. In 2006, Onur received bachelor degree on biomedical engineering at Başkent University Faculty of Engineering. Meanwhile, he was accepted to the Biomedical Engineering MSc program at Istanbul University Graduate School of Natural and Applied Science and started to work as biomedical engineer at Başkent University Istanbul Training and Research Hospital. In 2009, he resigned from the hospital for his compulsory military service. After finishing his military service in 2010, he joined to Medtronic Turkey Neuromodulation business unit as sales representative and received MSc degree from Istanbul University. In 2015, Onur was assigned as Deep Brain Stimulation Therapy Sales Manager by Medtronic Turkey and started his $\mathrm{PhD}$ studies in Maastricht University under supervision of Prof. Dr. Yasin Temel. Due to his personal interest in deep brain stimulation therapy and professional responsibilities, he provided intraoperative technical support to over one thousand deep brain stimulation surgeries and investigated technical aspects of DBS surgeries in various studies. He presented these studies at ESSFN congress in 2016 (Madrid / Spain) and WSSFN congress in 2017 (Berlin / Germany). By August 2018, Onur was assigned as Deep Brain Stimulation Therapy Senior Sales Manager by Medtronic Turkey. In March 2019, he joined Livanova Neuromodulation Business Unit as Field Clinical Manager and still continues his professional career in this role.

Onur married Didem Savaş Alptekin in 2018 and lives in Istanbul/Turkey with her. 